\title{
UHPLC-HRMS Analysis of Fagus sylvatica (Fagaceae) Leaves: A Renewable Source of Antioxidant Polyphenols
}

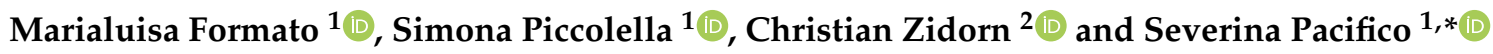 \\ 1 Department of Environmental, Biological and Pharmaceutical Sciences and Technologies (DiSTABiF), \\ University of Campania 'Luigi Vanvitelli', Via Vivaldi 43, 81100 Caserta, Italy; \\ marialuisa.formato@unicampania.it (M.F.); simona.piccolella@unicampania.it (S.P.) \\ 2 Department of Pharmaceutical Biology, Pharmaceutical Institute, Kiel University, Gutenbergstraße 76, \\ 24118 Kiel, Germany; czidorn@pharmazie.uni-kiel.de \\ * Correspondence: severina.pacifico@unicampania.it
}

Citation: Formato, M.; Piccolella, S.; Zidorn, C.; Pacifico, S. UHPLC-HRMS Analysis of Fagus sylvatica (Fagaceae) Leaves: A Renewable Source of Antioxidant Polyphenols. Antioxidants 2021, 10, 1140. https:// doi.org/10.3390/antiox10071140

Academic Editors: Filomena Nazzaro and Vincenzo De Feo

Received: 23 June 2021

Accepted: 16 July 2021

Published: 19 July 2021

Publisher's Note: MDPI stays neutral with regard to jurisdictional claims in published maps and institutional affiliations.

Copyright: (c) 2021 by the authors. Licensee MDPI, Basel, Switzerland. This article is an open access article distributed under the terms and conditions of the Creative Commons Attribution (CC BY) license (https:/ / creativecommons.org/licenses/by/ $4.0 /)$.

\begin{abstract}
European beech (Fagus sylvatica L.) is a deciduous tree, widely distributed in Europe and largely appreciated for its wood and nutritive nuts. Beech leaf also enjoys food use as salad, but an understanding of its nutraceutical value is still far from being achieved. Indeed, and also taking into account beech leaf as a consistent biomass residue available beechwood production and use, it needs to be explored as a valuable renewable specialized source of bioactive molecules. In this context, an untargeted ultra-high-performance liquid chromatography hyphenated with high resolution mass spectrometry (UHPLC-HRMS) approach was favorably applied to a beech leaf alcoholic extract, which also was evaluated for its antiradical capability (by means of assays based on 2,2-diphenyl-1picrylhydrazyl (DPPH) and [2,2'-azinobis-(3-ethylbenzothiazolin-6-sulfonic acid)] (ABTS) radical cation) and its ferric ion reducing power. Redox mitochondrial activity towards Caco-2 cells paved the way to explore the extract's capability to inhibit intracellular Reactive Oxygen Species (ROS) using $2^{\prime}, 7^{\prime}$ dichlorofluorescin diacetate (DCFH-DA) assay. Hydroxycinnamoyl derivatives, mainly belonging to the chlorogenic acid class, and flavonoids were the main constituents. Uncommon flavanone $C$-glycosides were also found, together with a plentiful flavonol diversity. Cell-free and cell-based assays highlight its dose-dependent antioxidant efficacy, providing a foundation for further investigation of beech leaf constituents and its valorization and use as a reservoir of bioactive natural products with potential nutraceutical applications.
\end{abstract}

Keywords: Fagus sylvatica L.; beech leaf; ultra-high-pressure liquid chromatography electroSpray ionization quadrupole time-of-flight mass spectrometry (UHPLC-ESI-QqTOF HRMS) analysis; antioxidant assays; polyphenol recovery

\section{Introduction}

Nowadays, the replacement of synthetic and artificial chemicals with natural products with less impact on human or animal health and environment, makes their recovery a major challenge. Indeed, great efforts have been devoted to the discovery and exploitation of renewable sources of valuable bioactive compounds, such as the so-called bioactive specialized natural products. These latter include a number of compound classes and sub-classes that, in the last years, have attracted a lot of attention for a possible application in various sectors (i.e., nutri-cosmeceutical, medical and pharmacological), due to their recognized benefits for human and animal health [1]. Thus, the research and valorization of new plant matrices, also those not directly used for these purposes, need to be explored as a virtuous source of these molecules. Innovative examples are agro-food wastes [2-4], or biorefining of forest biomass [5], from which compounds with traditional use in the prevention and/or treatment of different diseases could be favorably isolated [6]. Byproducts from forestry and wood processing industry are promising feedstocks for the 
extraction of polyphenols, including tannins and other antioxidants [7]. Thus, the selfrenewal of plant matrices such as the deciduous leaves of forest or ornamental trees, could be an alternative reservoir of compounds, exploitable for different useful applications.

Among forest trees, the Fagaceae family includes more than 900 species belonging to 8-10 genera, among which only Castanea, Fagus and Quercus are distributed in Asia, Europe and North America. In particular, Fagus sylvatica L., known as common or European beech, is an economically important tree species. It is widely distributed, from Northern Spain to Southern Italy and the North of Greece in the South Europe up to Southern Scandinavia in the North, and from the Atlantic Ocean in the West to the Black Sea in the East.

F. sylvatica leaves, as well as those of other forest trees, played an important role in winter-feeding of livestock in Europe even after haymaking replaced leaf-fodder harvesting over time. Indeed, different organs of Fagus sylvatica could be used as source of several natural products [8]. Thus, while beech bark and wood could be a source of different polyphenols [9], the fruits, named beechnuts, are used to obtain an oil rich in oleic and linoleic acids, as well as in $\gamma$ - and $\delta$-tocopherols [10]. Unlike wood, beechnuts or bark, whose chemical composition was rather well studied, there is very little literature data on the chemical composition of the leaves [11], which comprises phenols and polyphenols such as flavonoids, hydroxycinnamic acids and procyanidins [8,12], and triterpenes, such as glycosylated oleanane-type saponins [13]. Leaf ethanolic extracts showed antimicrobial activity [14], gastroprotective effects [15], with efficacy against Helicobacter pylori, and antiproliferative properties [16]. Beyond their chemical composition and biological activity, leaves are considered an index to evaluate environmental pollution and defensive mechanism to stress [17]. In the light of the above, the main objective of this study was to gain deep insights into the polyphenol composition of F. sylvatica leaves, in order to show the potential of their exploitation to obtain bioactive molecules. For this purpose, an untargeted UHPLC-HRMS (Ultra-High-Performance Liquid Chromatography-High-Resolution Mass Spectrometry) approach was applied to a methanolic leaf extract. The determination of total phenols and flavonoids, as well as the antiradical and reducing activity of the extract was also assessed, while its ability to slow down intracellular reactive oxygen species (ROS) formation was preliminarily investigated in Caco-2 cells.

\section{Materials and Methods}

\subsection{Plant Collection and Extraction}

Fagus sylvatica leaves were collected in June 2017 in the Tannenberger Gehölz (Kiel,

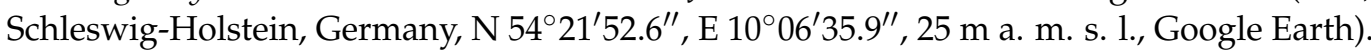
In particular, leaves harvested between ca. 0.5 and $2 \mathrm{~m}$ above ground were collected from six individual trees of various ages. The month average precipitation was $5 \mathrm{~mm}$ and average temperature was $21-10^{\circ} \mathrm{C}$ (max-min). Voucher specimens were deposited in the herbarium of Kiel University (KIEL, Schleswig-Holstein Land, Germany) and the private herbarium of Christian Zidorn (voucher code: FS_20160705A-1). Air-dried leaves $(240 \mathrm{~g})$ were shredded, and then extracted by maceration in methanol overnight, followed by an ultrasound accelerated maceration step (UAM; Bransonic ${ }^{\mathrm{TM}} \mathrm{M} 3800-\mathrm{E}$, Branson Ultrasonics $^{\mathrm{TM}}$, Danbury, CT, USA) (three sonication cycles, $35 \mathrm{~min}$ each). The alcoholic extract was stored at $-20{ }^{\circ} \mathrm{C}$ until use.

\subsection{UHPLC-HRMS and MS/MS Parameters}

The alcoholic extract was investigated using a NEXERA UHPLC system (Shimadzu, Tokyo, Japan) equipped with a Luna ${ }^{\circledR}$ Omega C-18 column $(50 \times 2.1 \mathrm{~mm}$ i.d., $1.6 \mu \mathrm{m}$ particle size). Two $\mu \mathrm{L}$ of the sample were injected. The mobile phase was constituted by water (solvent A) and acetonitrile (solvent B), both acidified with formic acid $(0.1 \% v / v)$. 
A linear gradient was used, in which the percentage of solvent $\mathrm{B}$ increased as follows: $0-5 \mathrm{~min}, 5 \% \rightarrow 15 \% \mathrm{~B} ; 5-10 \mathrm{~min}, 15 \% \mathrm{~B} ; 10-12 \mathrm{~min}, 15 \% \rightarrow 17.5 \% \mathrm{~B} ; 12-17 \mathrm{~min}, 17.5 \% \rightarrow 45 \% \mathrm{~B}$; 17-18.50 $\mathrm{min}, 45 \% \mathrm{~B} ; 18.51-20 \mathrm{~min}$, column re-equilibration. The flow rate was set at $400 \mu \mathrm{L} / \mathrm{min}$.

High-Resolution Mass Spectrometry (HR-MS) data were obtained by an AB SCIEX Triple TOF $^{\circledR} 4600$ mass spectrometer (AB Sciex, Concord, ON, Canada), equipped with a DuoSpray $^{\mathrm{TM}}$ ion source (AB Sciex, Concord, ON, Canada) operating in the negative ElectroSpray (ESI) mode. A full scan Time-Of-Flight (TOF) survey (accumulation time $100 \mathrm{~ms}, 100-1000 \mathrm{Da}$ ) and 8 information-dependent acquisition MS/MS scans (accumulation time $50 \mathrm{~ms}, 80-850 \mathrm{Da}$ ) were acquired, using the following parameters: curtain gas 35 psi, nebulizer and heated gases 60 psi, ion spray voltage $4500 \mathrm{~V}$, ion source temperature $600{ }^{\circ} \mathrm{C}$, declustering potential $-70 \mathrm{~V}$, collision energy $-35 \pm 5 \mathrm{~V}$. The instrument was controlled by Analyst ${ }^{\circledR}$ TF 1.7 software (AB Sciex, Concord, ON, Canada), whereas MS data were processed by PeakView ${ }^{\circledR}$ software version 2.2 (AB Sciex, Concord, ON, Canada). The compounds were identified mainly through the study of their tandem mass spectrometry (TOF-MS/MS) fragmentation patterns, and the comparison with literature data whenever possible.

\subsection{Radical Scavenging Capacity: DPPH and ABTS Tests}

Leaf alcoholic extract was tested at 200, 100, 50, 25, 12.5, 6.25, and $3.125 \mu \mathrm{g} / \mathrm{mL}$ (final concentration levels) towards ABTS [2,2'-azinobis-(3-ethylbenzothiazolin-6-sulfonic acid)] radical cation and 2,2-diphenyl-1-picrylhydrazyl (DPPH) radical.

ABTS radical cation was generated as previously reported [18]. The ABTS ${ }^{\bullet+}$ solution was diluted with Phosphate-buffered saline (PBS; pH 7.4) until an absorbance of 0.7 at $734 \mathrm{~nm}$ was read. The extract at different doses was directly dissolved in the ABTS ${ }^{\bullet+}$ solution, and after 6 min the absorbance was measured by a Victor3 spectrophotometer (Perkin Elmer/Wallac, Waltham, MA, USA) in reference to a blank, in which the samples were replaced with solvent [18].

$\mathrm{DPPH}^{\bullet}$ scavenging capability was estimated as previously reported [18], and the absorption at $517 \mathrm{~nm}$ was measured on the Victor3 spectrophotometer (Perkin Elmer/Wallac, Waltham, MA, USA) in reference to a blank, in which the samples were replaced with the solvent.

Trolox $(4,8,16,32 \mu \mathrm{M})$ was used as positive standard, and Trolox Equivalent Antioxidant Capacity (TEAC) of beech-leaf extract was calculated, based on both ABTS and DPPH tests. For each antiradical test, three replicate measurements for three samples $(n=3)$ of the extract (in total, $3 \times 3$ measurements) were performed. All data were expressed as mean \pm standard deviation (SD).

\subsection{Fe(III) Reducing Power}

Beech leaf alcoholic extract (at 200, 100, 50, 25, 12.5, 6.25, and $3.125 \mu \mathrm{g} / \mathrm{mL}$ final concentration levels) was investigated for its ability to reduce the $\mathrm{Fe}^{3+}$ using ferricyanide FRAP assay, as previously reported [19]. The absorbance was measured at $700 \mathrm{~nm}$. The increase in absorbance with reference to the blank was considered to value the reducing power. Trolox $(4,8,16,32 \mu \mathrm{M})$ was used as positive standard, and TEAC value of beech-leaf extract was calculated. The test was carried out performing three replicate measurements for three samples $(n=3)$ of the extract (in total, $3 \times 3$ measurements). All data were expressed as mean \pm standard deviation (SD). 


\subsection{Determination of Total Phenols}

Total phenol content was determined according to the Folin-Ciocalteau procedure [19]. Samples $(0.25 \mathrm{mg}$ and $0.125 \mathrm{mg})$ were mixed with $2.25 \mathrm{~mL}$ of $\mathrm{Na}_{2} \mathrm{CO}_{3}(7.5 \% \mathrm{w} / v)$ and $0.25 \mathrm{~mL}$ of Folin-Ciocalteu reagent. The tubes were mixed and allowed to stand for $3 \mathrm{~h}$ at room temperature. The absorbance was read at $765 \mathrm{~nm}$ using a Synergy spectrophotometer (Biotek, Winooski, VT, USA). The test was carried out performing three replicate measurements for three samples $(n=3)$ of the extract (in total, $3 \times 3$ measurements). Data were expressed as milligrams of gallic acid equivalents (GAEs) per $\mathrm{g}$ of extract (mean \pm standard deviation). To this purpose, a gallic acid calibration curve $\left(R^{2}=0.9716\right)$ was built up in the range $0.78-25 \mu \mathrm{g} / \mathrm{mL}$ (final concentration levels).

\subsection{Determination of Total Flavonoids}

The extract $(0.5 \mathrm{~mL})$ was dissolved in distilled water $(5 \mathrm{~mL})$, and $\mathrm{NaNO}_{2}$ solution $(5 \%, w / v ; 0.3 \mathrm{~mL})$ was added. After $5 \mathrm{~min}, \mathrm{AlCl}_{3}$ solution $(10 \%, w / v ; 0.6 \mathrm{~mL})$ was poured into the flask, and after $6 \mathrm{~min}, \mathrm{NaOH}$ solution $(1.0 \mathrm{M} ; 2.0 \mathrm{~mL})$ and distilled water $(2.1 \mathrm{~mL})$ were added. The absorbance was read at $510 \mathrm{~nm}$ against the blank (water), and flavonoid content is expressed as milligrams of quercetin equivalents per $100 \mathrm{~g}$ of fresh material [20]. To this purpose, a quercetin calibration curve $\left(R^{2}=0.9979\right)$ was built up in the range $0.78-100 \mu \mathrm{g} / \mathrm{mL}$ (final concentration levels). The test was carried out performing three replicate measurements for three samples $(n=3)$ of the extract (in total, $3 \times 3$ measurements). All data were expressed as mean \pm standard deviation (SD).

\subsection{Cell Culture, Cytotoxicity and Intracellular ROS Assessment}

Human epithelial cell line Caco-2 (ATCC ${ }^{\circledR} \mathrm{HTB} \neg 37^{\mathrm{TM}}$, American Type Culture, Manassas, VA, USA) was cultured in Dulbecco's Modified Eagle's Medium (DMEM) supplemented with $10 \%$ fetal bovine serum, $50.0 \mathrm{U} / \mathrm{mL}$ of penicillin and $100.0 \mu \mathrm{g} / \mathrm{mL}$ of streptomycin, at $37^{\circ} \mathrm{C}$ in a humidified atmosphere containing $5 \% \mathrm{CO}_{2}$. Cells were seeded in 96-multiwell plates at a density of $2.5 \times 10^{4}$ cells/well. After $24 \mathrm{~h}$, cells were treated for $24 \mathrm{~h}$ with different doses of the beech leaf alcoholic extract $(25,50,100$ and $200 \mu \mathrm{g} / \mathrm{mL})$ or pure quercetin standard $(5,10$, and $50 \mu \mathrm{M})$. When incubation was completed, inhibition of mitochondrial redox activity was determined by the MTT cell viability test, which was based on the 3-(4,5-dimethylthiazol-2-yl)-2,5-diphenyltetrazolium bromide (MTT) dye, as previously described [3,4]. In order to evaluate intracellular ROS inhibition [21], Caco-2 cells were seeded at a density of $2.5 \times 10^{4}$ well on a black 96-well microplate in $100 \mu \mathrm{L}$ growth medium/well. Cells were cultured for $24 \mathrm{~h}$ at $37^{\circ} \mathrm{C}$ in $5 \% \mathrm{CO}_{2}$ and observed under the inverted phase contrast microscope. After $24 \mathrm{~h}$, the growth medium was removed, and cells were twice washed with PBS $(100 \mu \mathrm{L})$. Then, the cells were co-exposed to the investigated extract $(25,50,100$ and $200.0 \mu \mathrm{g} / \mathrm{mL}$; final concentration levels) or quercetin (10 $\mu \mathrm{M}$ ) and $2^{\prime}, 7^{\prime}$-dichlorofluorescin diacetate (DCFH-DA; $60 \mu \mathrm{M}$ ) for $60 \mathrm{~min}$. The treatment medium was then removed, the cells were washed with PBS, and 2,2'-azobis(2-methyl propionamidine)dihydrochloride (AAPH, $500 \mu \mathrm{M}$; $100 \mu \mathrm{L}$ ) was added. The 96-well microplate was placed into a PerkinElmer Victor3 Multilabel Plate Reader at $37^{\circ} \mathrm{C}$. The fluorescence intensity was measured at $485 \mathrm{~nm}$ excitation and $535 \mathrm{~nm}$ emission wavelength every $20 \mathrm{~min}$ for $100 \mathrm{~min}$. Two independent experiments were carried out performing in each six replicate measurements for three samples $(n=3)$ of the extract (in total, $6 \times 3$ measurements). Data were expressed as mean \pm standard deviation (SD). 


\section{Results and Discussion}

\subsection{Chemical Composition of F. sylvatica Leaf Methanolic Extract}

Nowadays, the growing consciousness of environmental sustainability promotes the recovery of waste from production chains, also as basis and foundation of a circular economy. Renewable forest materials are rich in nutrients and bioactive molecules, whose recovery opens up to the development of functional products with high added value [22], to be used in various production sectors, from food, to nutraceuticals, cosmetics, up to the creation of packaging. In this context, getting insight into the chemical composition of beech leaf, its diversity in polyphenols could represent its intrinsic value.

The analytical determination of the diversity in bioactive molecules, after suitable extraction of the matrix, represents the crucial step of the entire process. Actually, techniques in tandem mass spectrometry hyphenated with chromatographic separation methods allows the chemical composition to be finely unraveled through an untargeted approach that provides accuracy in phenolomic data and compounds identification [23].

In the present study UHPLC-ESI-HRMS and High-Resolution tandem mass spectrometry (HR-MS/MS) techniques were first applied to unravel the chemical composition of this undervalued plant source [24-26]. An untargeted metabolic approach was used. Sixty-nine compounds were tentatively identified (Figure 1), mainly belonging to phenol and polyphenol classes. These latter have been described also as constituents of other forest trees and their wastes. Indeed, recently, bulk samples of bark waste from Pinus contorta, Pinus sylvestris, and Quercus robur, were investigated in depth for their polyphenol content [27], and various biomass residues (shavings, edged cuts, and pruning wastes) from walnut were analyzed as sources of antioxidant compounds by means of a green extraction process [28]. In this context, Pycnogenol ${ }^{\circledR}$ (PYC) and Flavangenol ${ }^{\circledR}$ are good examples of commercially available pine bark-based products [29]. The bark of douglas fir (Pseudotsuga menziesii Franco), one of the premier timber trees in the world, was found a rich source in taxifolin, which is broadly applied in pharmaceutical preparations [30].

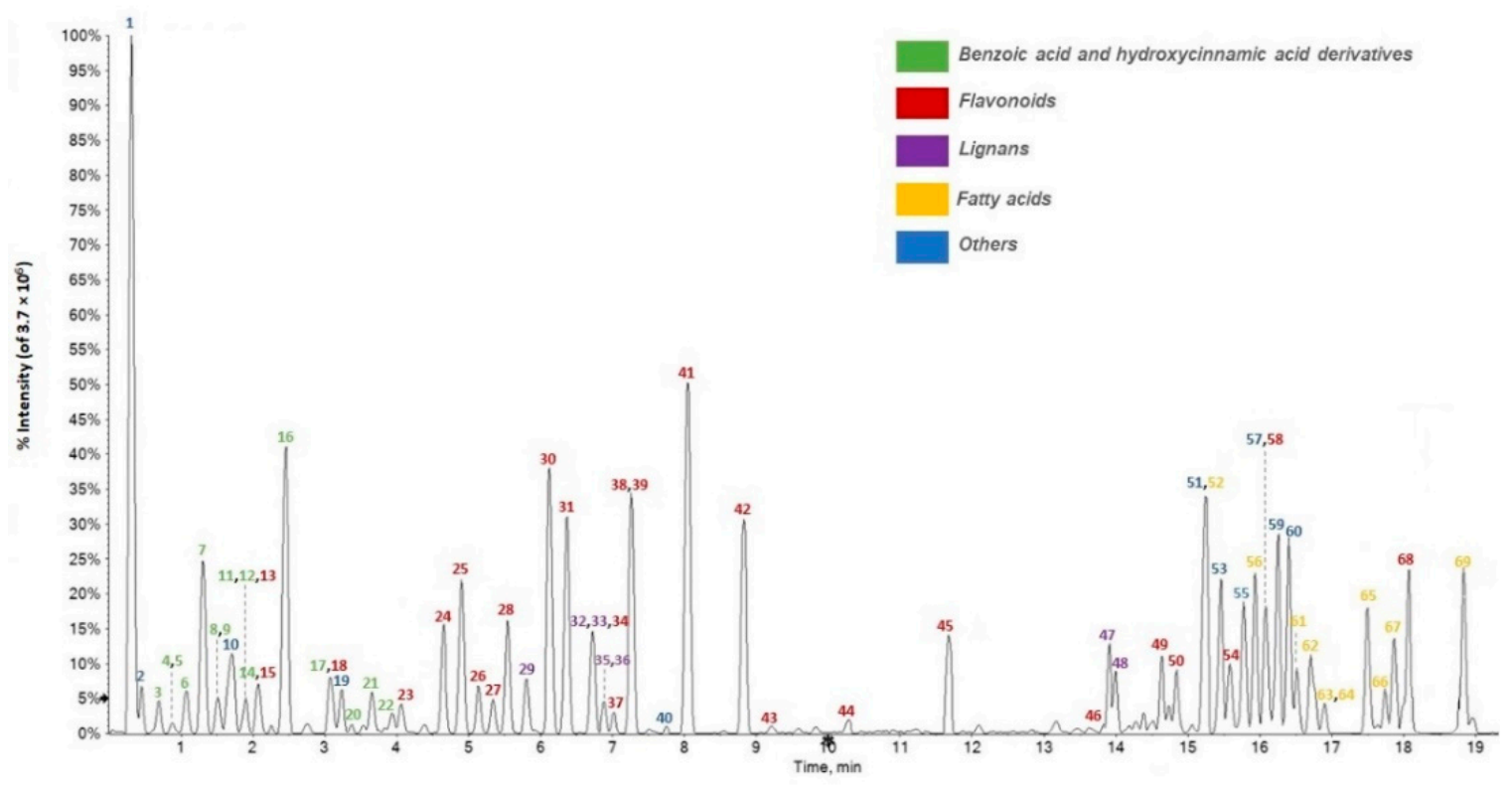

Figure 1. UHPLC-ESI-Q $q$ TOF MS profile (Total Ion Chromatogram acquired in negative ion mode) of $F$. sylvatica leaf extract under study. Compounds were numbered, based on their increasing retention time (RT). 
In Table 1, ESI negative ion mode MS and MS/MS data, molecular formulas, unsaturation degree (RDB-Ring and Double Bond) values and mass accuracy are listed.

\subsubsection{Benzoic and Hydroxycinnamic Acids Derivatives}

Compounds 4, 5 and 6 were tentatively identified as (di)hydroxybenzoic acid hexosides and dihydroxybenzoic acid, respectively. In fact, deprotonated glycosides underwent homolytic and heterolytic cleavages of the hexose moiety, providing fragment ions at $\mathrm{m} / \mathrm{z}$ 153.0191 and $m / z 152.0112$.

Compounds 7 and 16, previously identified in F. sylvatica leaves, were tentatively identified, in a ratio 1:2, as 3-O- and 5-O-caffeoyl quinic acid (Figure 2, panels A and B) based on the elution order and different fragmentation patterns [31]. The fragment ion at $m / z 179.03$ (deprotonated caffeic acid) was also identified in TOF-MS/MS spectra of compounds 8 (at $m / z$ 341.0879), 9 (at $m / z$ 297.0613) and 17 (at $m / z$ 253.0718), which were tentatively identified as caffeoyl acid hexoside $\left(\mathrm{C}_{15} \mathrm{H}_{18} \mathrm{O}_{9}\right)$, caffeoyl threonic acid $\left(\mathrm{C}_{13} \mathrm{H}_{14} \mathrm{O}_{8}\right)$ and caffeoyl propanoic acid $\left(\mathrm{C}_{12} \mathrm{H}_{14} \mathrm{O}_{6}\right)$, respectively. The fragmentation pattern of metabolite 9 showed also product ions at $m / z 135.0304$ and $m / z$ 117.0192 (Figure 2D), corresponding to deprotonated threonic acid and its dehydrated derivative. Instead, for metabolite $\mathbf{1 7}$ the propionyl moiety $\left(\mathrm{C}_{3} \mathrm{H}_{6} \mathrm{O}_{2}\right)$ was identified by neutral loss of $74.04 \mathrm{Da}$ (Figure 2E). Other caffeoyl derivatives were the metabolites 3, tentatively identified as hydroxycaffeoyl quinic acid, and 12, that putatively corresponded to a dimer of caffeoyl quinic acid. In particular, in the TOF-MS/MS spectrum of metabolite 3, whose isomer, with antimicrobial activity against Staphylococcus aureus and Escherichia coli, was isolated from Hymenocrater calycinus (Boiss.) Benth. [32], the deprotonated molecular ion at $m / z 371.1052$ underwent neutral loss of $18 \mathrm{Da}$, providing the less intense ion at $m / z 353.0872$ (caffeoyl quinate), and gave rise to the abundant ion at $m / z 191.0554$ (quinate) (Figure 2F). Deprotonated molecular ion of metabolite 12, at $m / z$ 707.1845, underwent neutral loss of $174 \mathrm{Da}$ and $192 \mathrm{Da}$, whose presence was further confirmed by fragment ion at $m / z$ 191.0557. Moreover, according to TOF-MS/MS spectrum, the ring arrangement could be of the $\beta$-truxillic acid type; cleavages along the two axes of central core provided the fragment ions at $m / z 463.1085,353.0870$ and 243.0651 (Figure S1).

Furthermore, coumaroyl derivatives were also identified: compounds 11 and 20, at $m / z$ 337.0921(18), were tentatively identified as 3-O- and 5-O-p-coumaroyl quinic acid ( $p$ CoQAs) in accordance with the molecular formula $\mathrm{C}_{16} \mathrm{H}_{18} \mathrm{O}_{9}$ (Figure 2,panels $\mathrm{G}$ and $\mathrm{H}$ ), whereas compound 14, showing the $[\mathrm{M}-\mathrm{H}]^{-}$ion at $m / z 325.0921$, was tentatively identified as $p$-coumaroyl hexoside (Figure 2I). In all TOF-MS/MS spectra, the fragment ion at $m / z$ 163.04 was detected, highlighting the presence of deprotonated coumaric acid $\left(\mathrm{C}_{9} \mathrm{H}_{8} \mathrm{O}_{3}\right)$. Compounds 21 and 22, whose deprotonated molecular ion was in accordance with the molecular formula $\mathrm{C}_{16} \mathrm{H}_{16} \mathrm{O}_{8}$, could be caffeoylshikimic acids (CSAs) [31]. Both metabolites showed fragment ions at $m / z 179.03$ and 161.02, generated by neutral loss of $156 \mathrm{Da}$ (shikimic acid- $\mathrm{H}_{2} \mathrm{O}$ ) and $174 \mathrm{Da}$ (shikimic acid) (Figure 2, panels J and K). The intensity of this latter allowed us to identify compound 22 as 4-O-CSA. 


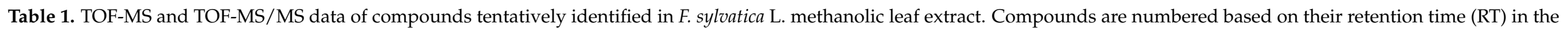
total ion current chromatogram ( $\mathrm{RDB}=$ Ring Double Bond equivalent value).

\begin{tabular}{|c|c|c|c|c|c|c|c|c|}
\hline Peak & $\begin{array}{c}\text { RT } \\
\text { (Min) }\end{array}$ & Tentative Assignment & Formula & $\begin{array}{c}{[\mathbf{M}-\mathbf{H}]^{-}} \\
\text {Calc. } \\
(m / z)\end{array}$ & $\begin{array}{c}{[\mathbf{M}-\mathbf{H}]^{-}} \\
\text {Found } \\
(m / z)\end{array}$ & $\begin{array}{r}\text { Error } \\
(\mathrm{ppm})\end{array}$ & RDB & MS/MS Fragment Ions $(\mathrm{m} / \mathrm{z})$ and Relative Intensity \\
\hline \multicolumn{9}{|c|}{ Benzoic and hydroxycinnamic acid derivatives } \\
\hline 3 & 0.699 & Hydroxycaffeoyl quinic acid & $\mathrm{C}_{16} \mathrm{H}_{20} \mathrm{O}_{10}$ & 371.0984 & 371.0970 & -3.7 & 7 & $\begin{array}{l}\text { 371.1052(3.4); 353.0872(3.4); 341.0900(3.4); 191.0554(100); } \\
\text { 173.0467(5.3); 135.0447(5.3) }\end{array}$ \\
\hline 4 & 0.875 & Dihydroxybenzoic acid hexoside & $\mathrm{C}_{13} \mathrm{H}_{16} \mathrm{O}_{9}$ & 315.0719 & 315.0722 & -0.8 & 6.0 & $\begin{array}{l}\text { 315.0714(18.8); 153.0191(16.0); 152.0112(60.5); 109.0294(34.8); } \\
\text { 108.0217(100) }\end{array}$ \\
\hline 5 & 1.034 & Hydroxybenzoic acid hexoside & $\mathrm{C}_{13} \mathrm{H}_{16} \mathrm{O}_{8}$ & 299.0772 & 299.0769 & -1.1 & 6 & $\begin{array}{l}299.0780(29.3) ; 239.0572(17.8) ; 179.0341(41.4) ; 137.0242(58.6) ; \\
136.0159(17.8) ; 121.0290(100) ; 93.0345(41.4)\end{array}$ \\
\hline 6 & 1.073 & Dihydroxybenzoic acid & $\mathrm{C}_{7} \mathrm{H}_{16} \mathrm{O}_{4}$ & 153.0193 & 153.0198 & 3.1 & 5 & 109.0291(100); 108.0215(87.5); 91.0185(12.4); 81.0341(8.7) \\
\hline 7 & 1.226 & 3-O-Caffeoyl quinic acid & $\mathrm{C}_{16} \mathrm{H}_{18} \mathrm{O}_{9}$ & 353.0878 & 353.0893 & 0.8 & 8 & $191.0565(100) ; 179.0354(48.1) ; 135.0453(44.5) ; 134.0370(5.8)$ \\
\hline 8 & 1.495 & Caffeoyl acid hexoside & $\mathrm{C}_{15} \mathrm{H}_{18} \mathrm{O}_{9}$ & 341.0874 & 341.0878 & -1.2 & 7 & $\begin{array}{l}\text { 341.0886(9.4); } 179.0339(22.0) ; 161.0244(100) ; 135.0444(9.4) \\
89.0243(18.6)\end{array}$ \\
\hline 9 & 1.514 & Caffeoyl threonic acid & $\mathrm{C}_{13} \mathrm{H}_{14} \mathrm{O}_{8}$ & 297.0616 & 297.0613 & -0.1 & 7 & 179.0344(9.6); 135.0304 (100); 117.0192 (6.6); 89.0246(11.6) \\
\hline 11 & 1.921 & 3-O-p-Coumaroyl quinic acid & $\mathrm{C}_{16} \mathrm{H}_{18} \mathrm{O}_{8}$ & 337.0929 & 337.0921 & -2.3 & 8 & 191.0550(61.4);173.0493(4.5);163.0396(100); 119.0946(74.6) \\
\hline 12 & 1.921 & Caffeoyl quinic acid dimer & $\mathrm{C}_{32} \mathrm{H}_{36} \mathrm{O}_{18}$ & 707.1829 & 707.1831 & 0.3 & 15 & $\begin{array}{l}707.1845(100) ; 533.1307(2.3) ; 515.1192(3.8) ; 463.1085(3.5) ; \\
353.0870(8.2) ; 323.0546(3.2) ; 243.0651(2.6) ; 191.0557(32.1)\end{array}$ \\
\hline 14 & 2.058 & $p$-Coumaroyl acid hexoside & $\mathrm{C}_{15} \mathrm{H}_{18} \mathrm{O}_{8}$ & 325.0929 & 325.0921 & -2.4 & 7 & $163.0394(46.2) ; 119.0499(100)$ \\
\hline 20 & 3.576 & 5-O-p-Coumaroyl quinic acid & $\mathrm{C}_{16} \mathrm{H}_{18} \mathrm{O}_{8}$ & 337.0929 & 337.0918 & -3.2 & 8 & $\begin{array}{l}\text { 191.0560(100); 173.0442(4.0); 163.0405(6.0); 119.0491(6.7); } \\
\text { 93.0346(16.9); 87.0073(3.7); 85.0290(2.7) }\end{array}$ \\
\hline 21 & 3.929 & 5-O-Caffeoyl shikimic acid & $\mathrm{C}_{16} \mathrm{H}_{16} \mathrm{O}_{8}$ & 335.0772 & 335.0773 & -0.4 & 9 & $\begin{array}{l}\text { 335.0755(3.9); } 179.0346(61.5) ; 173.0454(2.3) ; 161.0239(24.5) \\
\text { 135.0446(100); } 134.0372(6.9) ; 93.0344(6.9)\end{array}$ \\
\hline 22 & 4.047 & 4-O-Caffeoyl shikimic acid & $\mathrm{C}_{16} \mathrm{H}_{16} \mathrm{O}_{8}$ & 335.0772 & $\begin{array}{l}355.0768 \\
\text { Flavonoids }\end{array}$ & -1.3 & 9 & $335.0748(3.9) ; 179.0350(21.5) ; 161.0237(100) ; 135.0449(22.7)$ \\
\hline 13 & 1.921 & Procyanidin (B type) & $\mathrm{C}_{30} \mathrm{H}_{26} \mathrm{O}_{12}$ & 577.1352 & 577.1355 & 0.6 & 18 & $\begin{array}{l}577.1366(22.0) ; 451.1030(14.9) ; 425.0863(27.3) ; 407.0772(100) ; \\
381.0998(12.6) ; 299.0523(11.1) ; 289.0710(79.4) ; 245.0444(18.5) ; \\
125.0240(79.4)\end{array}$ \\
\hline 15 & 2.136 & Catechin & $\mathrm{C}_{15} \mathrm{H}_{14} \mathrm{O}_{6}$ & 289.0718 & 289.0708 & -3.3 & 9 & $\begin{array}{l}289.0696(38.3) ; 245.0818(30.8) ; 221.0797(30.8) ; 205.0505(15.0) ; \\
203.0709(54.1) ; 187.0381(30.8) ; 179.0365(23.3) ; 151.0403(40.6) ; \\
137.0246(30.8) ; 125.0238(30.8) ; 123.0447(84.2) ; 109.0290(100)\end{array}$ \\
\hline
\end{tabular}


Table 1. Cont.

\begin{tabular}{|c|c|c|c|c|c|c|c|c|}
\hline Peak & $\begin{array}{c}\text { RT } \\
\text { (Min) }\end{array}$ & Tentative Assignment & Formula & $\begin{array}{c}{[\mathrm{M}-\mathrm{H}]^{-}} \\
\text {Calc. } \\
(\mathrm{m} / \mathrm{z})\end{array}$ & $\begin{array}{c}{[\mathbf{M}-\mathbf{H}]^{-}} \\
\text {Found } \\
(\mathrm{m} / \mathrm{z})\end{array}$ & $\begin{array}{l}\text { Error } \\
(\mathrm{ppm})\end{array}$ & RDB & MS/MS Fragment Ions $(\mathrm{m} / \mathrm{z})$ and Relative Intensity \\
\hline 18 & 3.048 & Eriodictyol 7-O-hexoside & $\mathrm{C}_{21} \mathrm{H}_{22} \mathrm{O}_{11}$ & 449.1089 & 449.1097 & 1.7 & 11 & $\begin{array}{l}\text { 449.1097(2.5); 421.1142(8.5); 313.0717(2.5); 301.0709(9.4); } \\
\text { 287.0553(33.9); 259.0609(100); 243.0661(8.2) }\end{array}$ \\
\hline 23 & 4.125 & Kaempferol 3,7 di-O-hexoside & $\mathrm{C}_{27} \mathrm{H}_{30} \mathrm{O}_{16}$ & 609.1461 & 609.1466 & 0.8 & 13 & $\begin{array}{l}\text { 609.1517(100); 489.1064(25.2); 447.0941(82.9); 446.0886(49.6); } \\
285.0390(82.9) ; 284.0300(25.2) ; 283.0241(28.1)\end{array}$ \\
\hline 24 & 4.637 & Naringenin $8-C$-hexoside (1) & $\mathrm{C}_{21} \mathrm{H}_{22} \mathrm{O}_{10}$ & 433.1140 & 433.1145 & 1.1 & 11 & $\begin{array}{l}\text { 433.1165(7.1); 343.0830(34.3); 313.0724(100); 271.0616(15.9); } \\
\text { 223.0251(6.7); 193.0142(24.0); 119.0503(5.4) }\end{array}$ \\
\hline 25 & 4.917 & Naringenin $8-C$-hexoside (2) & $\mathrm{C}_{21} \mathrm{H}_{22} \mathrm{O}_{10}$ & 433.1140 & 433.1145 & 1.1 & 11 & $\begin{array}{l}433.1146(6.2) ; 343.0819(30.6) ; 313.0714(100) ; 271.0603(14.8) \\
223.0243(5.6) ; 193.0136(24.3) ; 165.0817(4.8) ; 119.0501(9.1)\end{array}$ \\
\hline 26 & 5.149 & Myricetin 3-O-hexoside & $\mathrm{C}_{21} \mathrm{H}_{20} \mathrm{O}_{13}$ & 479.0831 & 479.0844 & 12 & 2.7 & $\begin{array}{l}\text { 479.0855(32.8); 317.0300(14.7); 316.0226(100); 287.0193(6.1); } \\
\text { 271.0239(11.1) }\end{array}$ \\
\hline 27 & 5.772 & Myricetin 3-O-pentoside & $\mathrm{C}_{20} \mathrm{H}_{18} \mathrm{O}_{12}$ & 449.0725 & 449.0746 & 4.6 & 12 & $\begin{array}{l}\text { 449.0728(26.4); 317.0287(7.5); 316.0216(100); 287.0183(10.5); } \\
\text { 271.0241(18.4); }\end{array}$ \\
\hline 28 & 5.812 & Kaempferol 3-O-dihexoside & $\mathrm{C}_{27} \mathrm{H}_{30} \mathrm{O}_{16}$ & 609.1461 & 609.1481 & 3.3 & 13 & 609.1481(28.6); 285.0408(69.3); 284.0320(100); 255.0289(7.3) \\
\hline 30 & 6.046 & Quercetin 3-O-hexoside & $\mathrm{C}_{21} \mathrm{H}_{20} \mathrm{O}_{12}$ & 463.0882 & 463.0893 & 2.4 & 12 & $\begin{array}{l}\text { 463.0902(21.1); 301.0352(60.3); 300.0274(100); 271.0246(28.6); } \\
\text { 255.0292(15.9) }\end{array}$ \\
\hline 31 & 6.223 & Quercetin 3-O-hexuronide & $\mathrm{C}_{21} \mathrm{H}_{18} \mathrm{O}_{13}$ & 477.0675 & 477.0692 & 3.6 & 13 & $\begin{array}{l}\text { 477.0694(5.5); 301.0354(100); 283.0237(2.5); 255.0295(2.3); } \\
\text { 178.9974(6.3); 151.0029(6.2) }\end{array}$ \\
\hline 37 & 7.024 & $\begin{array}{l}\text { Isorhamnetin hexosyl } \\
\text { deoxyhexoside }\end{array}$ & $\mathrm{C}_{28} \mathrm{H}_{32} \mathrm{O}_{16}$ & 623.1618 & 623.1642 & 3.9 & 13 & $\begin{array}{l}\text { 623.1636(100); 315.0501(11.1); 314.0423(70.7); 299.0176(15.2); } \\
\text { 285.0423(5.4); 271.0256(5.4) }\end{array}$ \\
\hline 38 & 7.196 & Quercetin 3-O-pentoside & $\mathrm{C}_{20} \mathrm{H}_{18} \mathrm{O}_{11}$ & 433.0776 & 433.0795 & 4.3 & 12 & $\begin{array}{l}\text { 433.0796(16.5); 301.0355(19.2); 300.0281(100); 271.0247(21.7); } \\
\text { 255.0295(10.4) }\end{array}$ \\
\hline 39 & 7.240 & $\begin{array}{l}\text { Kaempferol } \\
\text { 3-O-galactopyranoside }\end{array}$ & $\mathrm{C}_{21} \mathrm{H}_{20} \mathrm{O}_{11}$ & 447.0933 & 447.0946 & 2.9 & 12 & $\begin{array}{l}\text { 447.0944(39.4); 327.0500(2.6); } 285.0397(27.2) ; 284.0322(100) \\
\text { 255.0293(40.7); } 227.0342(23.5)\end{array}$ \\
\hline 41 & 8.003 & Kaempferol 3-O-glucopyranoside & $\mathrm{C}_{21} \mathrm{H}_{20} \mathrm{O}_{11}$ & 447.0933 & 447.0943 & 2.3 & 12 & $\begin{array}{l}447.0943(35.5) ; 285.0398(55.2) ; 284.0322(100) ; 255.0295(47.0) \\
227.0345(27.7)\end{array}$ \\
\hline 42 & 8.897 & Kaempferol 3-O-pentoside & $\mathrm{C}_{20} \mathrm{H}_{18} \mathrm{O}_{10}$ & 417.0827 & 417.0844 & 4.0 & 12 & $\begin{array}{l}\text { 417.0858(31.8); 285.0410(22.1); 284.0337(100); 255.0306(53.1); } \\
\text { 227.0355(31.5) }\end{array}$ \\
\hline 43 & 9.203 & Kaempferol (acetyl)-hexoside & $\mathrm{C}_{23} \mathrm{H}_{22} \mathrm{O}_{12}$ & 489.1039 & 489.1054 & 3.2 & 13 & $\begin{array}{l}\text { 489.1071(36.3); 285.0383(37.7);284.0313(100); 255.0307(28.4); } \\
227.0329(12.3)\end{array}$ \\
\hline
\end{tabular}


Table 1. Cont.

\begin{tabular}{|c|c|c|c|c|c|c|c|c|}
\hline Peak & $\begin{array}{l}\text { RT } \\
\text { (Min) }\end{array}$ & Tentative Assignment & Formula & $\begin{array}{l}{[\mathbf{M}-\mathbf{H}]^{-}} \\
\text {Calc. } \\
(m / z)\end{array}$ & $\begin{array}{c}{[\mathbf{M}-\mathbf{H}]^{-}} \\
\text {Found } \\
(m / z)\end{array}$ & $\begin{array}{r}\text { Error } \\
(\mathrm{ppm})\end{array}$ & RDB & MS/MS Fragment Ions $(\mathrm{m} / \mathrm{z})$ and Relative Intensity \\
\hline 44 & 10.260 & Kaempferol 7-O-pentoside & $\mathrm{C}_{20} \mathrm{H}_{18} 0_{10}$ & 417.0827 & 417.0833 & 3.3 & 12 & $\begin{array}{l}\text { 417.0833(38.1); 285.0398(100); 284.0330(85.6); 255.0301(52.1); } \\
227.0355(38.1)\end{array}$ \\
\hline 45 & 11.672 & Kaempferol 7-O-deoxyhexoside & $\mathrm{C}_{21} \mathrm{H}_{20} \mathrm{O}_{10}$ & 431.0984 & 431.0999 & 3.5 & 12 & $\begin{array}{l}\text { 431.1006(24.2); } 285.0407(100) ; 284.0328(87.5) ; 255.0299(44.6) ; \\
\text { 227.0349(20.6) }\end{array}$ \\
\hline 46 & 13.891 & Kaempferol (acetyl)-pentoside & $\mathrm{C}_{22} \mathrm{H}_{20} \mathrm{O}_{11}$ & 459.0933 & 459.0938 & 1.1 & 13 & $\begin{array}{l}\text { 459.0970(60.6); 285.0395(10.4); 284.0331(100); 255.0304(36.7); } \\
\text { 227.0350(26.1) }\end{array}$ \\
\hline 49 & 14.626 & $\begin{array}{l}\text { Kaempferol } \\
\text { p-coumaroyl-hexoside (1) }\end{array}$ & $\mathrm{C}_{30} \mathrm{H}_{26} \mathrm{O}_{13}$ & 593.1301 & 593.1326 & 4.3 & 18 & $\begin{array}{l}\text { 593.1359(81.4); 447.0956(6.9); 285.0408(100); 284.0332(52.3); } \\
\text { 255.0297(10.0); 227.0346(4.1) }\end{array}$ \\
\hline 50 & 14.747 & $\begin{array}{l}\text { Kaempferol } \\
\text { p-coumaroyl-hexoside (2) }\end{array}$ & $\mathrm{C}_{30} \mathrm{H}_{26} \mathrm{O}_{13}$ & 593.1301 & 593.1331 & 4.3 & 18 & $\begin{array}{l}593.1349(100) ; 447.0958(9.1) ; 307.0820(6.2) ; 285.0404(97.7) \\
284.0313(57.1) ; 255.0288(9.3)\end{array}$ \\
\hline 54 & 15.612 & $\begin{array}{l}\text { Kaempferol } p \text {-coumaroyl } \\
\text { Pentoside }\end{array}$ & $\mathrm{C}_{29} \mathrm{H}_{24} \mathrm{O}_{12}$ & 563.1195 & 563.1219 & 4.3 & 18 & $563.1230(69.8) ; 285.0406(100) ; 284.0316(52.3)$ \\
\hline 58 & 16.202 & $\begin{array}{l}\text { Luteolin } \\
\text { p-coumaroyl-deoxyhexoside }\end{array}$ & $\mathrm{C}_{30} \mathrm{H}_{26} \mathrm{O}_{12}$ & 577.1352 & 577.1378 & 4.6 & 18 & $\begin{array}{l}\text { 577.1384(11.9); 431.1034(1.8); 284.0333(6.1); 285.0409(100); } \\
\text { 283.0223(1.8); 257.0469(2.7); 229.0514(2.3) }\end{array}$ \\
\hline 68 & 18.075 & $\begin{array}{l}\text { Kaempferol di- } p \text {-coumaroyl } \\
\text { deoxyhexoside }\end{array}$ & $\mathrm{C}_{39} \mathrm{H}_{32} \mathrm{O}_{14}$ & 723.1719 & 723.1752 & 4.5 & 24 & $\begin{array}{l}\text { 723.1763(2.5); 577.1391(14.5); 559.1232(8.1); 437.1261(48.6); } \\
\text { 397.1358(4.8); 285.0404(100); 284.0322(19.1);273.0759(5.7); } \\
\text { 187.0395(8.6); 163.0400(19); 145.0295(4.9) }\end{array}$ \\
\hline 29 & 5.812 & Isolariciresinol hexoside & $\mathrm{C}_{26} \mathrm{H}_{34} \mathrm{O}_{11}$ & 521.2028 & 521.2046 & 3.4 & 10 & $\begin{array}{l}\text { 359.1504(2.6); 329.1396(100); 192.0791(3.9); 193.0833(2.8); } \\
\text { 175.0760(5.8); 160.0519(3.0) }\end{array}$ \\
\hline 32 & 6.722 & $\begin{array}{l}\text { Neolignan-9'-O- rhamnoside } \\
\text { isomer } 1\end{array}$ & $\mathrm{C}_{25} \mathrm{H}_{34} \mathrm{O}_{11}$ & 509.2028 & 509.2053 & 4.1 & 9 & $\begin{array}{l}\text { 509.2044(16.9); 491.1926(13.3); 473.1824(27.9); 461.1813(25.9); } \\
\text { 367.1395(53.6); 339.1450(6.2); 313.1290(98.3); 179.0712(100); } \\
\text { 167.0711(7.2); 161.0611(12.4); 149.0608(27.6); 147.0445(13.3); } \\
\text { 134.0373(8.9); 103.0405(8.0) }\end{array}$ \\
\hline 33 & 6.722 & $\begin{array}{l}\text { Cinchonain-I } \\
\text { isomer } 1\end{array}$ & $\mathrm{C}_{42} \mathrm{H}_{20} \mathrm{O}_{9}$ & 451.1035 & 451.1046 & 2.5 & 15 & $\begin{array}{l}451.1083(9.6) ; 341.0669(100) ; 299.0551(27.9) ; 297.0762(11.7) \\
\text { 281.0460(11.7);217.0131(7.5); 189.0186(15.0); 177.0185(18.2); } \\
161.0246(6.4)\end{array}$ \\
\hline 35 & 6.848 & $\begin{array}{l}\text { Cinchonain-I } \\
\text { isomer } 2\end{array}$ & $\mathrm{C}_{42} \mathrm{H}_{20} \mathrm{O}_{9}$ & 451.1035 & 451.1047 & 2.5 & 15 & $\begin{array}{l}\text { 451.1056(6.3); 341.0674(100); 299.0593(4.7); 297.0762(6.4); } \\
\text { 281.0451(12.5); 279.0652(4.7); 231.0288(6.3); 217.0136(9.3); } \\
\text { 189.0178(12.5); 177.0193(12.5); 161.0246(4.7) }\end{array}$ \\
\hline
\end{tabular}


Table 1. Cont.

\begin{tabular}{|c|c|c|c|c|c|c|c|c|}
\hline Peak & $\begin{array}{c}\text { RT } \\
\text { (Min) }\end{array}$ & Tentative Assignment & Formula & $\begin{array}{l}{[\mathbf{M}-\mathbf{H}]^{-}} \\
\text {Calc. } \\
(m / z)\end{array}$ & $\begin{array}{c}{[\mathbf{M}-\mathbf{H}]^{-}} \\
\text {Found } \\
(m / z)\end{array}$ & $\begin{array}{l}\text { Error } \\
(\mathrm{ppm})\end{array}$ & RDB & MS/MS Fragment Ions $(\mathrm{m} / \mathrm{z})$ and Relative Intensity \\
\hline 36 & 6.888 & $\begin{array}{l}\text { Neolignan-9'-O- rhamnoside } \\
\text { isomer } 2\end{array}$ & $\mathrm{C}_{25} \mathrm{H}_{34} \mathrm{O}_{11}$ & 509.2028 & 509.2051 & 4.4 & 9 & $\begin{array}{l}\text { 509.2071(4.1); 491.1961(18.1); 473.1830(29.1); 461.1830(25.6); } \\
\text { 458.1611(6.6); 367.1398(25.2); 313.1299(66.2); 179.0713(100); } \\
\text { 167.0704(6.6); 163.0607(14.0); 147.0443(3.4); 149.0609(26.8); } \\
146.0374(18.9) ; 103.0374(6.6)\end{array}$ \\
\hline 47 & 13.891 & $\begin{array}{l}4,9,9^{\prime} \text {-Trihydroxy-3, } 3^{\prime}, 5^{\prime} \\
\text {-trimethoxy-8-O-4' } \\
\text {-neolignan-7-O-deoxyhexoside } \\
9^{\prime} \text {-Hydroxy-7'-propen- } 3^{\prime}, 5^{\prime} \text { - }\end{array}$ & $\mathrm{C}_{27} \mathrm{H}_{38} \mathrm{O}_{12}$ & 553.2304 & 553.2321 & 5.5 & 9 & $\begin{array}{l}\text { 553.2291(6.1); 343.1382(100); 328.1140(19.2); 211.0595(2.1); } \\
\text { 183.0647(1.5) }\end{array}$ \\
\hline 48 & 14.017 & $\begin{array}{l}\text { dimethoxyphenyl-3- } \\
\text { methoxyphenyl-7,9-propanediol- } \\
\text { 4-O-hexoside }\end{array}$ & $\mathrm{C}_{27} \mathrm{H}_{38} \mathrm{O}_{12}$ & 551.2134 & 551.2167 & 6.0 & 10 & $\begin{array}{l}\text { 551.2184(4.7); 533.2083(4.7); 343.1394(14.1); 328.1161(4.7); } \\
\text { 209.0915(100); 194.0579(31.1); 176.0481(8.1) }\end{array}$ \\
\hline \multicolumn{9}{|c|}{ Fatty acids } \\
\hline 52 & 15.278 & Trihydroxy-octadecadienoic acid & $\mathrm{C}_{18} \mathrm{H}_{32} \mathrm{O}_{5}$ & 327.2177 & 327.2185 & 2.5 & 3 & $\begin{array}{l}\text { 327.2187(88.3); 309.2087(3.0); 291.1962(16.4); 229.1448(51.1); } \\
\text { 221.1183(15.4); 211.1341(100); 209.1186(7.9); 183.1385(27.6); } \\
\text { 171.1026(33.2) }\end{array}$ \\
\hline 56 & 16.067 & \multirow{2}{*}{$\begin{array}{l}\text { Hydroxyhexadecanoic acid } \\
\text { Dihydroxyoctadecedienoic quinic } \\
\text { acid } \\
\text { Linolenic acid derivative }\end{array}$} & $\mathrm{C}_{16} \mathrm{H}_{32} \mathrm{O}_{4}$ & 287.2228 & 287.2233 & 1.8 & 1 & $287.2236(100) ; 285.2077(7.2) ; 269.2114(8.8)$ \\
\hline 62 & 16.708 & & $\mathrm{C}_{31} \mathrm{H}_{38} \mathrm{O}_{6}$ & 505.2596 & 505.2589 & -1.3 & 13 & 505.2605(37.2); 277.2176(100); 227.0323(5.2); 152.9955(9.2) \\
\hline 63 & 16.864 & Hydroxyoctadecatrienoic acid & $\mathrm{C}_{18} \mathrm{H}_{30} \mathrm{O}_{3}$ & 293.2122 & 293.2129 & 2.3 & 4 & $\begin{array}{l}\text { 293.2111(65.6); 275.2014(82.9); 221.1539(59.0); 211.1338(25.2); } \\
\text { 183.1385(100); 171.1016(50.3) }\end{array}$ \\
\hline 64 & 16.883 & Linoleic acid derivative & $\mathrm{C}_{34} \mathrm{H}_{44} \mathrm{O}_{9}$ & 595.2913 & 595.2915 & 0.4 & 13 & $\begin{array}{l}595.2940(100) ; 415.2266(6.3) ; 315.0492(9.5) ; 279.2332(43.1) \\
241.0109(17.2) ; 152.9952(11.5)\end{array}$ \\
\hline 65 & 17.484 & $\begin{array}{l}\text { 15,16-Dihydroxy-9,12- } \\
\text { octadienoic } \\
\text { acid }\end{array}$ & $\mathrm{C}_{18} \mathrm{H}_{32} \mathrm{O}_{4}$ & 311.2228 & 311.2232 & 1.3 & 3 & $\begin{array}{l}311.2238(39.4) ; 293.2125(12.6) ; 275.2017(9.9) ; 235.1710(11.1) \\
\text { 253.1804 (3.4); } 223.1707(100) ; 183.0116(2.8)\end{array}$ \\
\hline 66 & 17.738 & $\begin{array}{l}\text { Linolenic acid } \\
\text { glyceryl-tetrahexoside }\end{array}$ & $\mathrm{C}_{45} \mathrm{H}_{76} \mathrm{O}_{24}$ & 999.4654 & 999.4685 & 3.1 & 8 & $\begin{array}{l}\text { 999.4751(100); 837.4302(2.0); 739.2582(12.1); 721.2452(29.1); } \\
559.1919(3.4) ; 397.1370(4.0) ; 221.0682(1.3) ; 119.0353(1.3)\end{array}$ \\
\hline 67 & 17.885 & Linolenic acid derivative & $\mathrm{C}_{32} \mathrm{H}_{41} \mathrm{NO}_{4}$ & 502.2963 & 502.2963 & 0.0 & 13 & 502.2958(17.3); 456.1546(6.3); 277.2175(100); 224.0689(6.3) \\
\hline 69 & 18.826 & $\begin{array}{l}\text { Linolenic acid } \\
\text { glyceryl-dihexoside }\end{array}$ & $\mathrm{C}_{33} \mathrm{H}_{56} \mathrm{O}_{4}$ & 675.3597 & 675.3629 & 4.7 & 6 & $\begin{array}{l}\text { 675.3622(13.0); 415.1454(43.5); 397.1344(100); 379.1210(2.7); } \\
\text { 305.0848(7.8); 277.2158(68.9); 253.0904(5.2); 235.0804(18.3), } \\
\text { 179.0557(5.2); 161.0451(3.5); 119.0342(7.8); 89.0247(7.8) }\end{array}$ \\
\hline
\end{tabular}


Table 1. Cont.

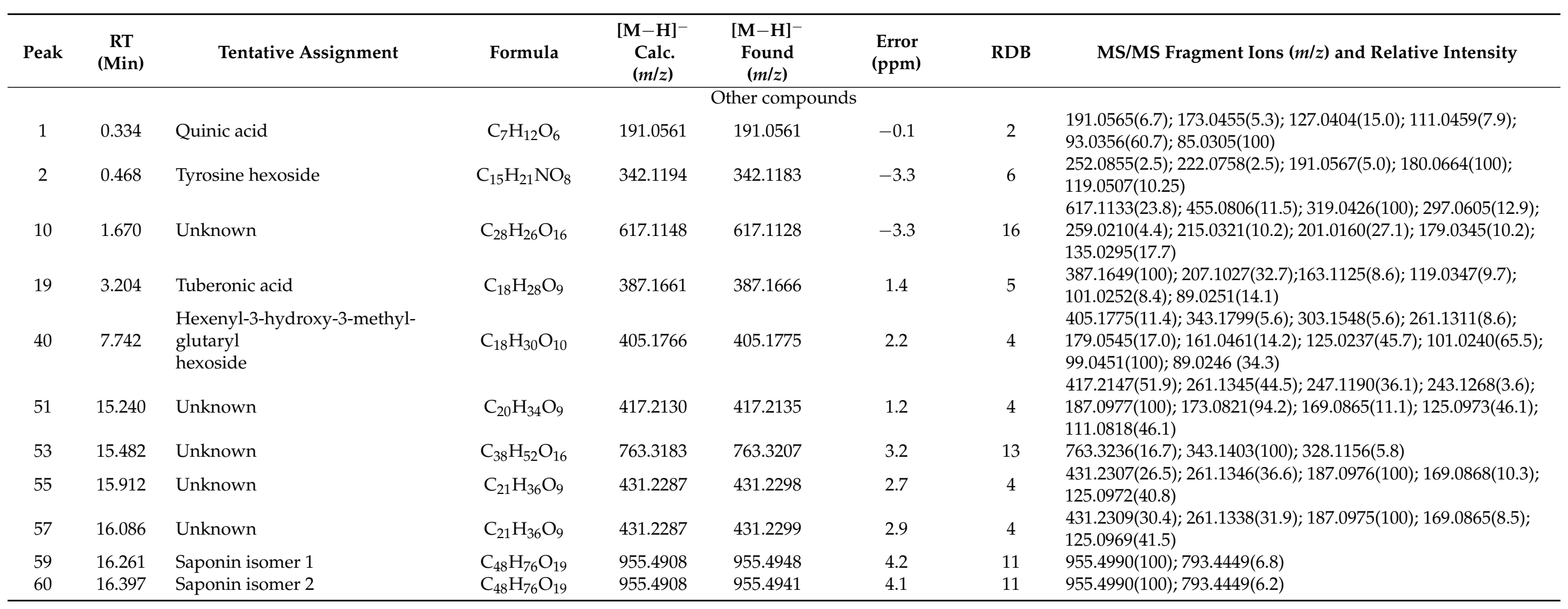



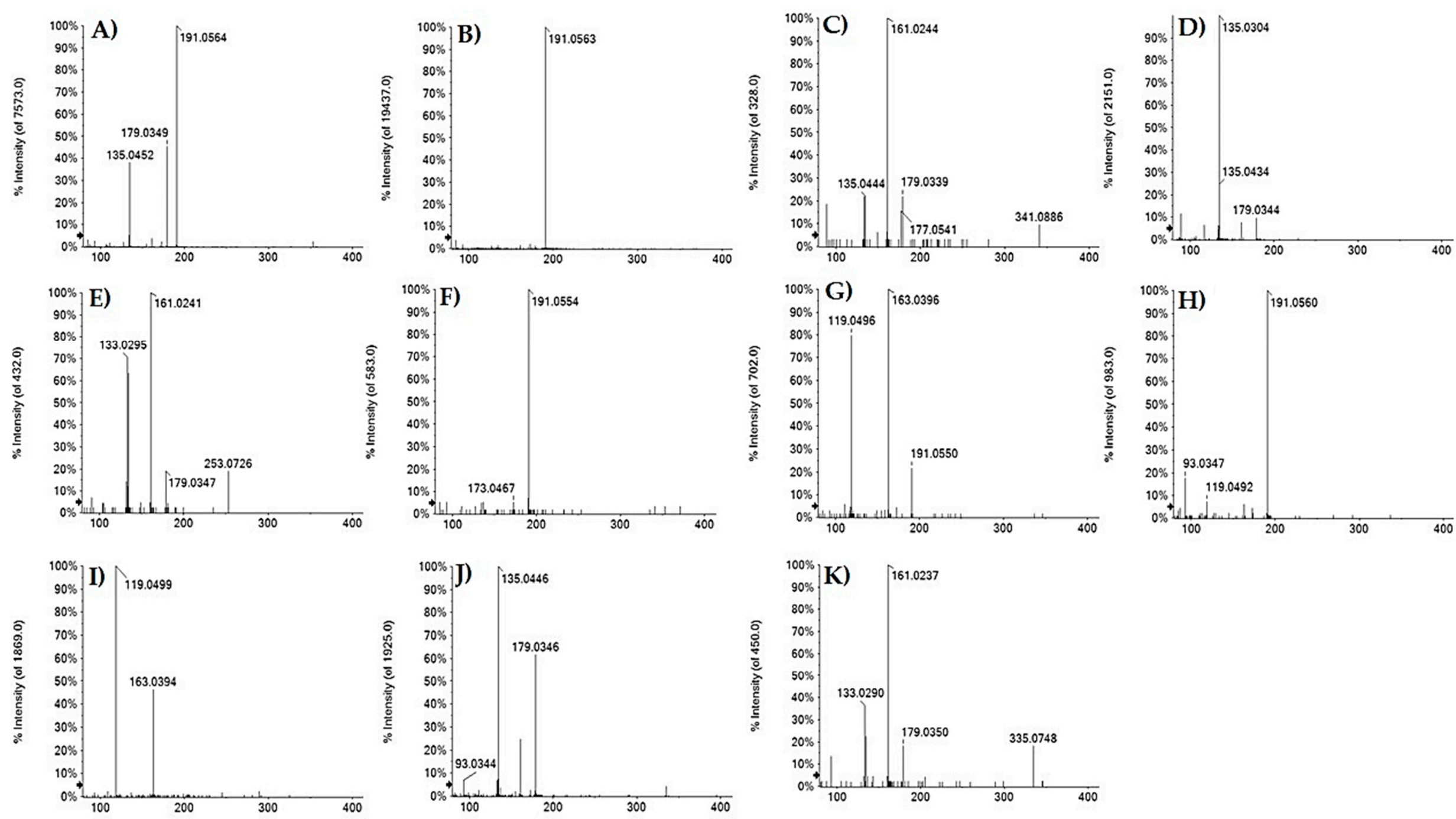

Figure 2. TOF-MS/MS spectra of deprotonated compounds (A) 7; (B) 16;(C) 8; (D) 9; (E) 17; (F) 3; (G) 11; (H) 20; (I) $14 ;($ J) $21 ;(\mathrm{K}) 22$. 


\subsubsection{Flavonoids}

Compounds 18, 24, 25, 34 were tentatively identified as flavanones. Compound 18 with the $[\mathrm{M}-\mathrm{H}]^{-}$ion at $m / z 449.1097$ was tentatively identified as eriodictyol-7-O-hexoside. In fact, the $[\mathrm{M}-\mathrm{H}]^{-}$ion provided in the TOF-MS/MS experiment an abundant deprotonated aglycone ion $(33.9 \%)$ at $m / z 287.0553$, following the neutral loss of a hexose moiety, and the fragment ion at $m / z 259.0609$ (base peak) by neutral loss of $\mathrm{CO}(28 \mathrm{Da}$ ) and hexose moiety (162.05 Da). Metabolites 24, 25 and 34 were hypothesized to be $C$-glycosylated flavanones, which are much less studied than O-glycosides, but endowed with several health benefits, such as antioxidant, anticancer, antitumor and anti-diabetic activities [33]. The occurrence of naringenin-C-glycosides was previously described by Hoffman et al. [25], but though an HPLC-MS/MS via Multiple Reaction Monitoring (MRM) analysis was performed, these authors did not provide sufficient MS/MS details to prove the presence of these compounds in beech leaves. The almost superimposable MS/MS fragmentation patterns were in accordance with naringenin $C$-hexoside isomers, likely bearing different sugar moieties that reasonably explained the different retention times (Figure 3). In fact, the deprotonated molecular ion at $m / z 433.11$ provided the ions $[\mathrm{Ag}+71]^{-}$at $\mathrm{m} / z 343.08$ and $[\mathrm{Ag}+41]^{-}$at $m / z 313.07$ (base peak) by the ${ }^{0,2} \mathrm{X}$ and ${ }^{0,3} \mathrm{X}$ cross-ring cleavage of hexoside, likely linked at C-8 position. This hypothesis was supported by very low intensity of dehydrated fragment ions at $m / z 415.10$ and 325.07, which are known to be much more pronounced for 6-C isomers [34]. Although the occurrence of this kind of compounds is quite unusual, some literature data consoled our hypothesis. Indeed, naringenin 8$C$ - $\beta$-glucopyranoside (isohemiphloin) was isolated from Eucalyptus hemiphloia F. Muell. (Myrtaceae), and its 6-C isomer (hemiphloin) was identified also in Ononis vaginalis M.Vahl. (Fabaceae), Tulipa gesneriana L. and Ulmus wallichiana Planch., beside eriodictyol 6-C- $\beta$-Dglucopyranoside [35].

Different flavan-3-ols mono- and diglycosides have been identified too. In Figure 4, neutral losses and related molecular formulas of their main fragmentations are schematized. In particular, considering flavonols group, monoglycosides were $82.2 \%$, diglycosides account $17.2 \%$, and (acyl)-glycoside flavonols were only $0.51 \%$. The deprotonated compounds $26(\mathrm{~m} / z 479.0844)$ and $27(\mathrm{~m} / z$ 449.0746) were putatively identified as myricetin 3-O-hexoside and myricetin 3-O-pentoside, respectively. In fact, the loss of $162 \mathrm{Da}$ (hexose moiety) and $132 \mathrm{Da}$ (pentose moiety) provided in both cases the fragment ion at $\mathrm{m} / \mathrm{z} 317.02$, attributable to myricetin, together with its aglycone radical anion at $m / z 316.02$, whose abundance allowed us to hypothesize the C-3 linkage of sugars moieties.

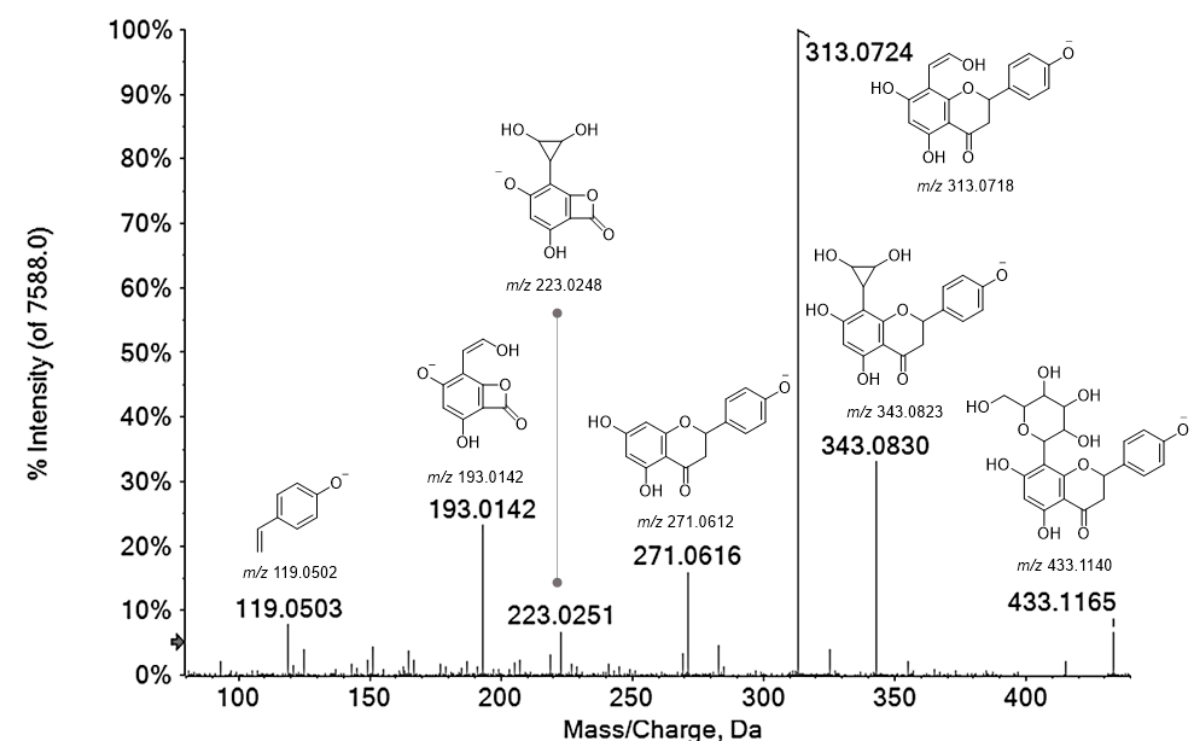

Figure 3. TOF-MS ${ }^{2}$ spectrum of metabolite 24. The chemical structure of each product ion is depicted, and theoretical $\mathrm{m} / \mathrm{z}$ ratio is indicated below. 


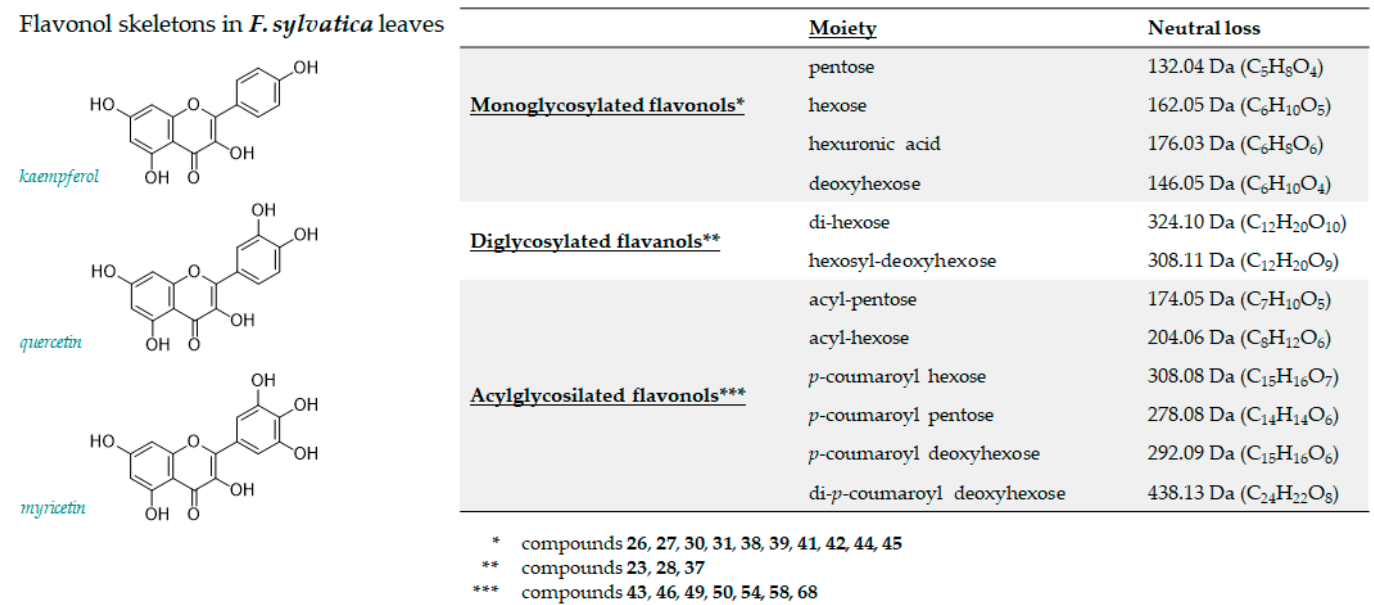

Figure 4. Chemical structures of major flavonol aglycones tentatively identified in the extract under study. Neutral losses with related molecular formulas have been reported to summarize and schematize the different moieties attached to aglycones.

Compounds 30, 31 and 38 shared quercetin as aglycone and they were identified as quercetin 3-O-hexoside (30), quercetin 3-O-hexuronide (31), and quercetin 3-O-pentoside (38), respectively $[3,11,31]$. Kaempferol glycosides appeared to be more abundant. Among them, metabolites $\mathbf{3 9}$ and $\mathbf{4 1}$ were putatively identified as 3-O-galactopyranoside and 3-O-glucopyranoside derivatives. These latter were more abundant among kaempferol derivatives, representing the $36.2 \%$. The shorter retention time of the 3-O-galactopyranoside derivative [36], the natural higher abundance of the 3-O-glucopyranoside isomer, and the relative intensity of fragment ion at $\mathrm{m} / \mathrm{z} 285$ allowed us to discriminate the two isomers. The mass spectrum of compound $\mathbf{4 2}$ was in accordance with kaempferol 3-O-pentoside. In fact, MS/MS spectrum of the deprotonated molecular ion at $m / z 417.0844$ generated, by neutral loss of $132 \mathrm{Da}$ (dehydrated pentose moiety), the $\left[\mathrm{M}-\mathrm{H}-\mathrm{C}_{5} \mathrm{H}_{9} \mathrm{O}_{4}\right]^{-}$ion at $\mathrm{m} / z$ 285.0410, whose corresponding radical aglycone ion at $m / z 284.0337$ was the peak base. Instead, the neutral loss of 204.06 Da allowed us to tentatively identify metabolite 43 (at $\mathrm{m} / \mathrm{z}$ 489.1054) as kaempferol (acetyl)-hexoside. Analogously, compound 46 at $m / z 459.0938$ was putatively identified as kaempferol (acetyl)-pentoside, whose glycosidic bond cleavage $(-174.05 \mathrm{Da})$ provided a low aglycone ion and an abundant radical aglycone ion. Finally, the different [aglycone- $\mathrm{H}]^{-} /$[aglycone-H] ${ }^{\bullet-}(m / z 285.0407 / 284.0328)$ ratio in the TOF$\mathrm{MS}^{2}$ spectrum of compound $\mathbf{4 5}$ was in accordance with a 7-O-deoxyhexosyl kaempferol, whereas, among flavanol diglycosides, compounds 23 and 28 , sharing the $[\mathrm{M}-\mathrm{H}]^{-}$ion at $m / z$ 609.1466(81) and the same molecular formula $\left(\mathrm{C}_{27} \mathrm{H}_{30} \mathrm{O}_{16}\right)$, were tentatively identified as kaempferol dihexosides.

Compound 37 showed a TOF-MS/MS spectrum in accordance with isorhamnetin hexosyl deoxyhexoside. In fact, the break of 3-O-glycosidic cleavage, with a loss of 308.11 Da (hexose+deoxyhexose), gave rise to the [aglycone- $\mathrm{H}]^{\bullet-}$ and [aglycone- $\left.\mathrm{H}\right]^{-}$ions at $\mathrm{m} / z$ 314.0423 and 315.0501, respectively. A further loss of $15 \mathrm{Da}$, providing fragment ions at $\mathrm{m} / \mathrm{z}$ 299.0176, supported the structural hypothesis. Metabolites 49, 50 and 68 were further identified as $p$-coumaroyl derivatives of kaempferol hexoside (49 and 50) and deoxyhexoside (68). The $[\mathrm{M}-\mathrm{H}]^{-}$ion at $m / z$ 593.1326(31) of the compounds 49 and 50 led to [aglycone$\mathrm{H}]^{-}$and [aglycone-H] ${ }^{\bullet-}$ ions by neutral loss of $308.0951 \mathrm{Da}$ and $309.1027 \mathrm{Da}$, in accordance with a $p$-coumaroylhexosyl moiety. In the TOF-MS/MS spectra the neutral loss of $146.04 \mathrm{Da}$, in accordance with the dehydrated $p$-coumaric acid, provided the ion at $m / z$ 447.0954(8), whose intensity allowed us to hypothesize the linkage of the hydroxycinnamic acid to the saccharide moiety. Instead, metabolite 68, tentatively identified as kaempferol di- $p$ coumaroyl deoxyhexoside, showed the $[\mathrm{M}-\mathrm{H}]^{-}$ion at $m / z 723.1752$ that dissociated giving fragment ions at $m / z 577.1391$ and 559.1232, according to neutral loss of dehydrated (or not) $p$-coumaric acid. The fragment ion at $m / z 437.1261$ (48.6\%) consisted in the acylated 
sugar moiety (di-p-coumaroyl-deoxyhexoside- $\mathrm{H}_{2} \mathrm{O}$ ), according to the observed neutral loss of $438.13 \mathrm{Da}$ from deprotonated molecular ion that, through heterolytic or homolytic cleavage, provided the ions at $m / z 285.0404$ and 284.0322. The deprotonated molecular ion for compound 58 (at $m / z$ 577.1378) was in accordance with a flavone acylglycoside, likely luteolin $p$-coumaroyl-deoxyhexoside. In the TOF-MS/MS spectrum, the $[\mathrm{M}-\mathrm{H}]^{-}$ion dissociated supplying the abundant fragment ion [aglycone- $\mathrm{H}]^{-}$at $m / z 285.0409$, through the neutral loss of $292.0975 \mathrm{Da}$ ( $p$-coumaric acid+deoxyhexose). The absence of fragment ions at $m / z 255$ and $m / z 227$, which allowed flavonols to be distinguished from flavones, allowed us to identify the aglycone as luteolin.

The fragmentation pattern of compound $\mathbf{1 3}$ was in accordance with the presence of procyanidin (B-type). In fact, the $[\mathrm{M}-\mathrm{H}]^{-}$ion at $m / z 577.1355$ dissociated losing the A-ring of the flavanolic unit ( $-126 \mathrm{Da}$ ) through the heterocyclic ring fission (HRF) mechanism, producing the fragment ion at $m / z 451.1030$. The fragment ion at $m / z 425.0863$ was due to retro-Diels Alder (RDA) mechanism, and the monomeric unit at $m / z 289.0710$ was generated through the quinone methide fission [37]. Moreover, the deprotonated molecular ion at $m / z 289.0708$ for compound $\mathbf{1 5}$ was in accordance with the molecular formula $\mathrm{C}_{15} \mathrm{H}_{14} \mathrm{O}_{6}$ and (epi)catechin compound. The decarboxylation ( $-44 \mathrm{Da})$ and subsequent loss of an ethenone unit $\left(\mathrm{C}_{2} \mathrm{H}_{2} \mathrm{O}\right)$, generated fragment ions at $m / z 245.0818$ and 203.0709, respectively; from the latter, the loss of lateral chain as 2-methylene-2H-pyran $\left(\mathrm{C}_{6} \mathrm{H}_{6} \mathrm{O} ;-94.04 \mathrm{Da}\right)$ for nucleophilic attack of hydroxyl group to benzylic carbon, provided base peak at $m / z$ 109.0290. Following the benzofuran-forming fission reaction from deprotonated molecular ion the ion at $m / z 123.0447$ was formed [37].

\subsubsection{Lignans}

Compounds 29, 32, 36, and 47 were tentatively identified as lignans. In particular, metabolite 29 was tentatively identified as isolariciresinol hexoside, whose deprotonated molecular ion at $m / z 521.2046$ underwent neutral loss of sugar moiety providing the fragment ion at $m / z 359.1504$, from which the loss of formaldehyde provided the abundant ion at $m / z$ 329.1396. Compounds 32 and 36 could be neolignan-O-deoxyhexoside isomers; these metabolites were previously reported as unidentified compounds [11]. Instead, two neolignan-9'-O-deoxyhexoside stereoisomers were isolated and identified by NMR in Fagus hayatae Palib. ex Hayata lea [38]. This finding is in line with the occurrence of these metabolites, which were also isolated in Pinus thunbergii [39]. Beside fragment ions at $m / z$ 491.1926(61) and 473.1824(30), which are derived from dehydration reactions, the bond cleavage between the two phenylpropanoid units provided fragment ions at $m / z$ 179.0712(13) (base peak) and 313.1290(99). In Figure S2 TOF-MS/MS spectra were reported, and the hypothesized chemical structures for the most abundant fragment ions were provided.

The metabolite 47, whose tentative characterization was possible with a slight modification of Q-TOF parameters (Figure S3), was identified as 4,9, $9^{\prime}$-trihydroxy-3,3',5'-8$O-4^{\prime}$-neolignan-7-O-deoxyhexoside [40]. The loss of $212.06 \mathrm{Da}$, likely corresponding to trimethoxygallic acid, generated the fragment ion at $m / z$ 343.1410, which in turn lost a methyl radical to generate the ion at $m / z$ 328.1177. The presence of the deoxyhexose moiety was confirmed by loss of $146.06 \mathrm{Da}$, providing the ion at $m / z$ 197.0821. As for compounds 32 and 36, the fragment ion derived by sugar loss has a very low intensity, likely justified by an intramolecular hydrogen bond. Compound 48 with the $[\mathrm{M}-\mathrm{H}]^{-}$ion at $m / z 551.2167$, was supposed to be a lignan (Figure S4): $9^{\prime}$-hydroxy-7'-propen-3', $5^{\prime}$-dimethoxyphenyl-3methoxyphenyl-7,9-propanediol-4-O-hexoside [41]. The fragment ion at $m / z 209.0815$ was attributable to a sinapyl alcohol moiety, from which ions at $m / z 194.0579$ and 179.0344 were generated. To the best of our knowledge, this lignan has never been identified in F. sylvatica.

Flavolignan compounds, such as compounds 33 and 35 (at $m / z$ 451.1047(46)) were tentatively identified. These compounds were likely cinchonain-I isomers, which are phenylpropanoid-substituted catechin, characterized by the ion at $m / z$ 341.0669(74), gener- 
ated from the loss of the 3,4-dihydroxyphenyl group ( $-110 \mathrm{Da}$ ). Subsequently, through the HRF mechanism and the RDA mechanism, the ions at $m / z$ 189.0186(78) and 177.0185(93) were formed, respectively.

\subsubsection{Fatty Acids}

The investigated extract also showed the occurrence of mono- or poly-hydroxylated fatty acids, whose presence, together with epoxy derivatives, was previously identified by Matzke et al. [42]. In particular, metabolite 52, was tentatively identified as trihydroxy octadecadienoic acid $(m / z$ 327.2185). It underwent dehydration processes, providing fragment ions at $m / z 309.2087$ and 291.1962. The C12-C13 bond cleavage provided the ion at $m / z 229.1448$, which in turn lost $58 \mathrm{Da}\left(\mathrm{C}_{3} \mathrm{H}_{6} \mathrm{O}\right)$ and $46 \mathrm{Da}$, to generate fragment ions at $m / z$ 183.1385 and 171.1026, respectively (Figure S5).

Compound 56 was putatively identified as hydroxyhexadecanoic acid, e.g., 16-OHhexadecanoic acid [33]), whose dehydration provided a low intensity ion at $m / z 269.2114$, whereas TOF-MS/MS spectrum of compound $\mathbf{6 1}$ indicated it could be dihydroxyoctadecedienoyl quinic acid. The loss of dehydrated quinic acid, identified by fragment ion at $m / z 191.0559$, produced an abundant ion at $m / z 311.2230$ that corresponded to the fatty acid. In addition to fragment ions at $m / z 293.2117$ and 275.2012, obtained by losses of hydroxy groups, also a fragment ion at $m / z 223.1703$, to cleavage between C-4 and C-5, was detected (Figure S6).

Compound 63 was putatively a hydroxyoctadecatrienoic acid, maybe with hydroxyl group at carbon C-10. In fact, the $\beta$-scission of the alcoholic group provided the ion $[\mathrm{M}-\mathrm{H}-$ 110] $]^{-}$at $m / z 183.1385$ identified as base peak. An allyl scission provided ions at $m / z$ 221.1539 and 211.1338 from dehydrated ion $(\mathrm{m} / z$ 275.2014) and deprotonated molecular ion, respectively. Compound 65 showed TOF-MS/MS spectrum in accordance with 15,16-dihydroxy-9,12-octadienoic acid [43]. Product ions deriving from water losses were detected at $m / z$ 293.2125 and 275.2017. Furthermore, allyl scission gave a low abundance ion at $m / z$ 253.1804, whereas the $\beta$-fission of the alcoholic hydroxyl group provided an abundant ion at $m / z$ 223.1707. Compound 69 (at $m / z$ 675.3629) was putatively identified as a linolenic acid glyceryl-dihexoside. In fact, the product ion at $m / z 277.2158$ corresponded to the fatty acid moiety, whereas glyceryl dihexoside could be identified by fragment ions at $m / z 415.1454$ and 397.1344; from the latter the loss of $92 \mathrm{Da}$, identifying a glycerol unit, provided the ion at $m / z$ 305.0848. In Figure S7, the TOF-MS/MS spectrum and a putative fragmentation pathway are reported.

\subsubsection{Other Minor Compounds}

None of the remaining compounds were assignable to any of the previously discussed classes. Briefly, compound $\mathbf{1}$ was likely quinic acid, whereas metabolite $\mathbf{2}$ was putatively identified as tyrosine hexoside, based on the presence of the ion at $\mathrm{m} / \mathrm{z} 180.0664$ (deprotonated tyrosine), formed after the neutral loss of the hexose moiety. Metabolite 19 with the $[\mathrm{M}-\mathrm{H}]^{-}$ion at $\mathrm{m} / z 387.1666$ was tentatively identified as 12 -hydroxyjasmonate (tuberonic acid) [18].

The TOF-MS/MS spectrum of compound 40 showed fragment ions at $m / z 125.0237$, 101.0240 and 99.0451 in accordance with a 3-hydroxy-3-methylglutaryl substitution of hexenyl hexoside [44]. Thus, the metabolite was putatively a 3-hydroxy-3-methylglutaryl(O-hexenyl) hexoside, from which the loss of $144 \mathrm{Da}(\mathrm{HMG})$ gave the fragment ion at $m / z$ 261.1311. Metabolites 59 and 60, with a $[\mathrm{M}-\mathrm{H}]^{-}$ion at $m / z$ 955.4948(41), were tentatively identified as triterpenoid saponins with oleanolic acid as aglycone, previously characterized in F. sylvatica leaves through spectroscopic techniques [13], but never by mass spectrometry analysis. In order to obtain useful information about the fragmentation pattern, declustering potential and collision energy values were optimized; thus, metabolite 59 was tentatively identified as 28-(D-glucopyranosyloxy)-28-oxoolean-12-en-3 $\beta$-yl-3-O-( $\beta$ D-glu-copyranosy1)- $\beta$-D-glucopyranosiduronic acid. The deprotonated molecular ion lost the hexosyl moiety, providing the ion at $m / z$ 793.4368(69), detected in TOF-MS/MS spectra 
of both metabolites, and underwent ${ }^{0,2} \mathrm{X}$ cross-ring cleavage of hexuronic acid ring, to give the ion at $m / z$ 659.4152, diagnostic for the identification of metabolite 60 [13,45] (Figure S8). The neutral loss of $176 \mathrm{Da}$ (dehydrated hexuronic acid) from ion at $\mathrm{m} / z 631.38$ provided the ion at $m / z$ 455.3505(16), most likely oleanolic acid.

\subsection{Relative Quantitation of F. sylvatica Leaf Chemical Constituents}

Hydroxycinnamic acid (HCA) derivatives constitute a considerable part of low molecular weight phenol compounds. Caffeoyl-based HCA compounds were the most abundant, as they account for $88.0 \%$ of the compounds with $\mathrm{C}_{6} \mathrm{C}_{1}$ and $\mathrm{C}_{6} \mathrm{C}_{3}$ carbon skeleton. In particular, chlorogenic acids, such as $3-\mathrm{O}$ - and 5 -O-caffeoyl quinic acid, were $27.9 \%$ and $54.9 \%$, respectively, whereas coumaroyl derivatives were less represented $(\sim 4.0 \%)$. The interest in chlorogenic acids is due to the plethora of beneficial effect ascribed to these substances, for which certain fruits, vegetables, spices are the main dietary sources. In particular, 5-O-caffeoyl quinic acid, firstly analyzed for its antioxidant, anti-inflammatory, and antitumor activity [46] was found to play multiple and key roles in protecting humans at neuronal, cardiovascular, and gastrointestinal levels. It was also implied in glucose and lipid metabolic regulation [47]. Besides caffeoyl derivatives, flavonoids appeared to be in appreciable amount. Flavonols were the main constituents of this class, with a relative percentage of $86.0 \%$. Figure 5 shows the relative content of benzoic/hydroxycinnamic acids class, and flavonoids.

Kaempferol derivatives were the most abundant $(66.0 \%)$, followed by quercetin derivatives $(24.0 \%)$, and myricetin derivatives $(10 \%)$. The abundance of flavonoids in different organs of $F$. sylvatica, such as leaves or bark $[12,48,49]$, was stated by different literature data, in which kaempferol, quercetin, myricetin, luteolin or naringenin derivatives were identified. Unusual naringenin-C-glycosides are as part of flavanone class, which is commonly associated to different benefits due to their ability to act as free radical-scavenger. Juice and peel of citrus fruits are the main dietary sources of these compounds [50], which were also clinically evaluated for cardiovascular disease protection. The anti-inflammatory, antioxidant and soothing effects of flavonoids are broadly exploited for food, drugs or cosmetics production [51].

A) benzoic and hydroxycinnamic acids

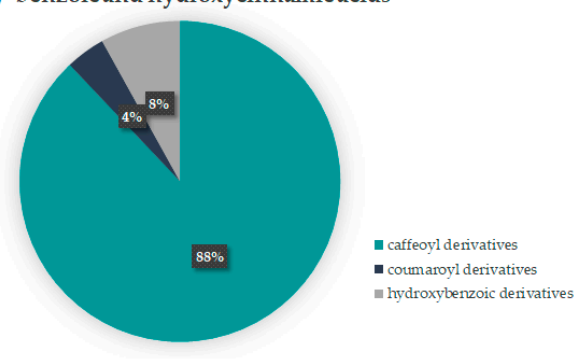

B) flavonoids

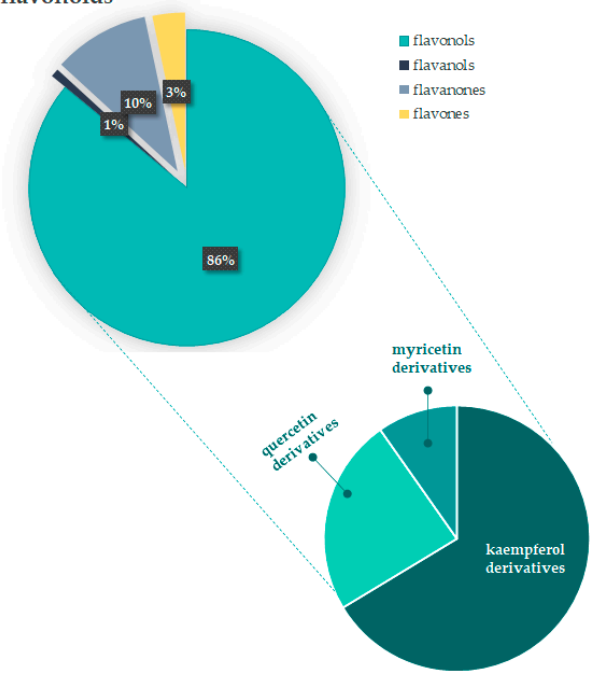

Figure 5. Semi-quantitative representation of beech leaf extract constituents. (A) Relative content of coumaroyl, caffeoyl and hydroxybenzoic derivatives into the class of benzoic and hydroxycinnamic acids; (B) Relative content of different flavonoid subfamilies.

Apart from antiradical and reducing activities, food-derived flavonoids were shown to prevent non-communicable diseases on-set, and to exert pro-oxidant effect in cancer cells, thus increasing ROS levels and apoptosis rate. Several epidemiological evidences 
suggest that kaempferol-rich foods reduce the risk of liver, colon and skin cancer [52], whereas the uncountable properties of quercetin and its derivatives give rise recently-filed patents for disparate therapeutic applications [53]. Towards a green and sustainable waste valorisation chain, the employment of $F$. sylvatica leaves should be pursued.

\subsection{Beech Leaf Alcoholic Extract Showed Antioxidant Efficacy in Cell-Free Assays}

The alcoholic leaf extract was evaluated for its antioxidant capability by means of assays, involving stable radicals, such as $\mathrm{DPPH}^{\bullet}$ and $\mathrm{ABTS}^{\bullet+}$ tests, and by ferricyanide FRAP assay. Folin-Ciocalteu test, which employs phospho-tungsto-molybdate, was also performed, together with the colorimetric determination of total flavonoid content. Data acquired showed that the extract was effective in scavenging ABTS ${ }^{\bullet+}$ with an $\mathrm{ID}_{50}$ and TEAC value equal to $31.4 \pm 1.10 \mu \mathrm{g} / \mathrm{mL}$ and 0.27 , respectively, and it was able to markedly reduce ferric ions, also at the lowest tested dose (Figure 6A). The total phenol content (TPC) was $69.64 \pm 3.1 \mathrm{mg}$ of gallic acid equivalents per g of extract (Figure 6B). Tanase et al. [24], who investigated TPC in beech bark hydroalcoholic extract, found it was $76.49 \mathrm{mg}$ GAE/g plant material. Comparably, bark extracts of F. sylvatica, obtained by means of different extractive methods were screened for their antiradical activity and phenol content, and their diversity in catechins, taxifolin glycosides, procyanidins, syringic acid or coniferyl alcohol glycosides were further estimated by chromatographic techniques hyphenated to mass spectrometry $[10,24,48,54]$. Among the few studies on beech leaf, a comparative qualitative study was carried out on hydroalcoholic extracts (EtOH:water, 7:3, v/v) of beech leaves collected in Romania; it was evidenced that TPC significantly varied based on leaf harvest times [12,55], reaching the maximum TPC value ( $33.55 \mathrm{mg} / \mathrm{g}$ GAE), when leaves were collected in September. According to UHPLC-ESI-Q qTOF analysis, a high content of flavonoid compounds was spectrophotometrically determined (Figure 6B).
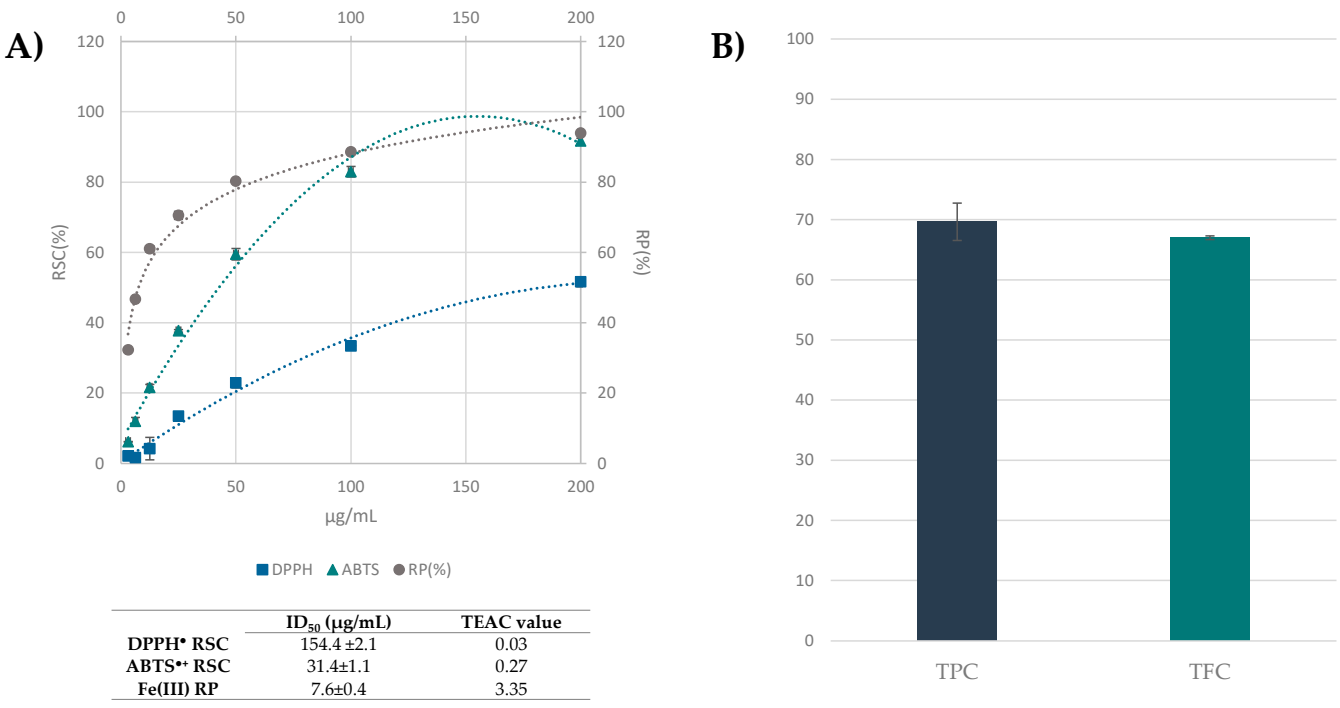

Figure 6. (A) Radical scavenging activity (RSC\%) of F. sylvatica leaf extract vs. 2,2-diphenyl-1picrylhydrazy (DPPH) radical (ם) and 2,2'-azino-bis(3-ethylbenzothiazoline-6-sulfonic acid (ABTS) radical cation $(\boldsymbol{\Lambda})$. Fe(III) Reducing Power $(\mathrm{RP} \%)$ is also measured $(\bullet)$. ID 50 values $(\mu \mathrm{g} / \mathrm{mL})$ and TEAC (Trolox Equivalent Antioxidant Capacity) values are tabulated. Values reported are the mean $\pm \mathrm{SD}$ of three independent measurements. (B) Total Phenol Content (TPC) were expressed as mg of gallic acid equivalent per $\mathrm{g}$ of extract, and Total Flavonoid Content (TFC) were expressed as mg of quercetin equivalent per $g$ of extract. Values reported are the mean $\pm \mathrm{SD}$ of three independent measurements.

\subsection{Beech Leaf Alcoholic Extract Decreased Intracellular ROS in Caco-2 Cells}

The extract richness in polyphenols and the positive responses of cell-free antioxidant tests paved the way to the evaluation of its antioxidant potential in cell-based assays. To this purpose, Caco-2 cell line represents a useful model, able to predict in vivo behavior. In fact, 
besides being widely used as an in vitro model for the intestinal epithelial barrier, they were reported to offer a distinctive advantage in screening intestinal absorption of natural antioxidants after oral intake [21]. Thus, with the aim to exploit beech leaf phytochemicals in the formulation of nutraceuticals, the ability of the extract to counteract reactive oxygen species (ROS) overproduction was preliminarily evaluated in Caco-2 cells [49]. In fact, metabolic activity is strongly related to ROS production, which intracellularly originate in the mitochondrial respiratory chain, also producing toxic metabolic byproducts [56]. Thus, redox mitochondrial activity was first assessed by means of MTT test (Figure 7A). The beech leaf alcoholic extract exhibited dose-response inhibition of the metabolic activity of Caco-2 cells, with an $\mathrm{ID}_{50}$ equal to $148.4 \mu \mathrm{g} / \mathrm{mL}$. In the cell model, after co-exposing Caco-2 cell monolayer to increasing dose levels of the extract and to the fluorescent probe DCFH-DA, oxidative stress is induced by 2,2-azobis(2-amidinopropane) dihydrochloride (AAPH). Based on fluorescence levels, which are related to the oxidation degree, it was observed that the extract markedly reduced the cellular fluorescence compared to the untreated cells, when it was tested at dose level equal to $200 \mu \mathrm{g} / \mathrm{mL}$ (Figure 7B). The other doses tested, while exhibiting less pronounced efficacy, showed a strongly time-dependent activity. Overall, the dose-dependent capability of the beech leaf extract was ascertained. Considering the cell basal rate in oxidizing species, the outcome of the investigated beech on redox balance could be due to extract-induced mitochondrial alterations. The effect was compared to that exerted by pure standard quercetin, which was tested at $10 \mu \mathrm{M}$, based on cell viability data and according to literature [21,57]. In fact, the peroxyl radical scavenging activity of quercetin in Caco-2 cells was broadly proved [21], and it was found that doses of the flavonol higher than $20 \mu \mathrm{M}$ markedly affected colon cells viability [57]

A)

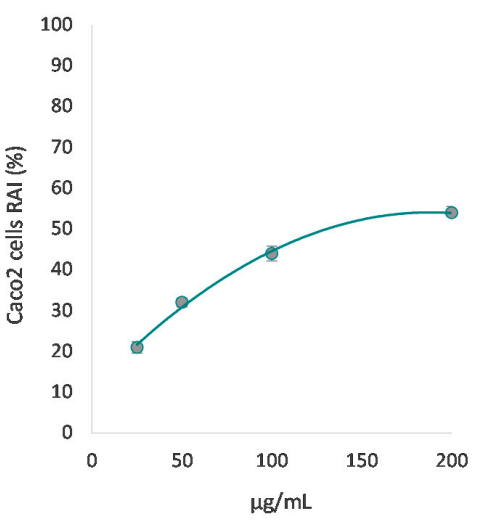

C)

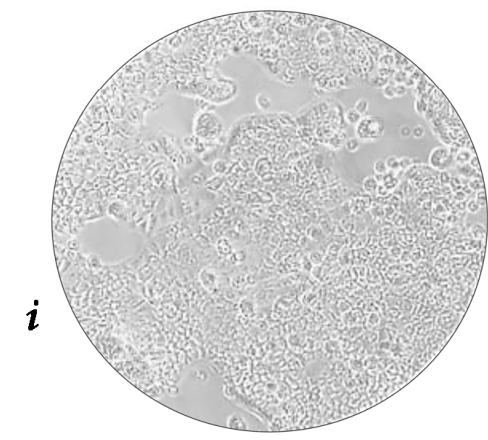

B)
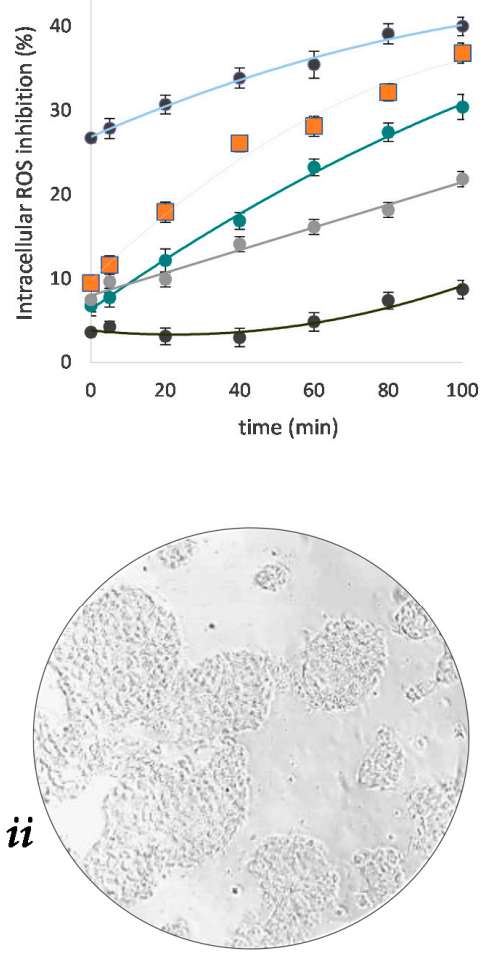

Figure 7. (A) Redox activity inhibition (RAI\%) of $F$. sylvatica leaf extract towards Caco-2 cells. (B) ROS intracellular inhibition (\%) of F. sylvatica leaf extract $\bullet 200 \mu \mathrm{g} / \mathrm{mL}, \bullet 100 \mu \mathrm{g} / \mathrm{mL}, \bullet 50 \mu \mathrm{g} / \mathrm{mL}$ - $25 \mu \mathrm{g} / \mathrm{mL}$, and of quercetin $10 \mu \mathrm{M}(\boldsymbol{\square})$. Values reported are the mean $\pm \mathrm{SD}$ of three independent measurements. (C) Representative images of untreated Caco-2 cells (i) and treated Caco-2 cells (ii) acquired by Inverted Phase Contrast Brightfield Zeiss Primo Vert Microscope. 


\section{Conclusions}

Fagus sylvatica leaves, investigated through an untargeted UHPLC-HR-MS/MS analysis, revealed their richness in flavonoids, mainly flavonol monoglycosides. Thus, the recovery of this renewable plant source could favor an application to create beech leaf-derived products, in the form of food or dietary supplements or herbal remedies for human or animal health. Cell-free assays evaluating the antiradical and reducing capability, as well as preliminary antioxidant capability in Caco- 2 cells, strengthened this exploitation hypothesis. Thus, the promising antioxidant results herein reported could be the basis for the further phytochemical investigation of $F$. sylvatica, and the use of different extractive and chromatographic approaches aimed at recovering high amounts of pure beech bioactive compounds.

Supplementary Materials: The following are available online at https:/ /www.mdpi.com/article/10 .3390/antiox10071140/s1, Figure S1: Proposed fragmentation pathway for compound 12, Figure S2: TOF-MS/MS spectra of the $[\mathrm{M}-\mathrm{H}]^{-}$ions of compounds 32 (A) and 36 (B), tentatively identified as neolignane- $9^{\prime}$-O-deoxyhexoside are reported with the structures of different fragment ions tentatively characterized. The proposed fragment pathway of much more abundant ions is also reported (C), Figure S3: MS/MS spectral differences of the $[\mathrm{M}-\mathrm{H}]^{-}$ions of compound 47 recorded at different Q-TOF parameters: CE $-75 \mathrm{~V}$, CES $25 \mathrm{~V}$ and DP $-90 \mathrm{~V}$ (A) or CE $-35 \mathrm{~V}$, CES $10 \mathrm{~V}$ and DP $-70 \mathrm{~V}$ (B). The proposed structures of more abundant ions are also reported, Figure S4: TOF-MS/MS spectrum of compound 48 , tentatively identified as $9^{\prime}$-hydroxy-7'-propen-3', $5^{\prime}$-dimethoxyphenyl-3methoxyphenyl-7,9-propanediol-4-O-hexoside and structures of different tentatively characterized fragment ions, Figure S5: TOF-MS/MS spectrum of metabolite 52, tentatively identified as trihydroxy octadecadienoic acid, whose hypothetical structure and fragmentation pathway was reported in the grey box (the theoretical $m / z$ value is reported below each structure), Figure S6: TOF-MS/MS spectrum of metabolite 61, tentatively identified as dihydroxyoctadecedienoic quinic acid, Figure S7: TOF-MS/MS spectrum of compound 69 . The proposed fragmentation pathway of its $[\mathrm{M}-\mathrm{H}]^{-}$ ion was reported in the grey box (the theoretical $\mathrm{m} / z$ value is reported below each structure), Figure S8: TOF MS/MS spectra of the $[\mathrm{M}-\mathrm{H}]^{-}$ions of metabolites 59 (A) and 60 (B); the proposed chemical structures of fragment ions much more abundant are reported with different neutral losses. The fragment ions detected only in one of two spectra are highlighted by red symbols.

Author Contributions: Conceptualization, S.P. (Severina Pacifico) and C.Z.; methodology, M.F., S.P. (Simona Piccolella), S.P. (Severina Pacifico); validation, M.F., S.P. (Simona Piccolella), S.P. (Severina Pacifico); formal analysis, M.F.; investigation, M.F.; resources, S.P. (Severina Pacifico); data curation, M.F.; writing — original draft preparation, M.F. and S.P. (Simona Piccolella); writing—review and editing, S.P. (Severina Pacifico); visualization, C.Z.; supervision, S.P. (Severina Pacifico); funding acquisition, S.P. (Severina Pacifico). All authors have read and agreed to the published version of the manuscript.

Funding: This research received no external funding.

Institutional Review Board Statement: Not applicable.

Informed Consent Statement: Not applicable.

Data Availability Statement: Data is contained within the article and supplementary material.

Acknowledgments: The study was supported by the grant "Programma Dottorato VALERE XXXV ciclo" from the University of Campania "Luigi Vanvitelli". The authors thank Friederike Scharenberg for European Beech collection, and Maria Tommasina Pecoraro for graphical abstract picture.

Conflicts of Interest: The authors declare no conflict of interest.

\section{References}

1. Newman, D.J.; Cragg, G.M. Natural Products as Sources of New Drugs over the Nearly Four Decades from 01/1981 to 09/2019. J. Nat. Prod. 2020, 83, 770-803. [CrossRef]

2. Ferri, M.; Bin, S.; Vallini, V.; Fava, F.; Michelini, E.; Roda, A.; Minnucci, G.; Bucchi, G.; Tassoni, A. Recovery of polyphenols from red grape pomace and assessment of their antioxidant and anti-cholesterol activities. New Biotechnol. 2016, 33, 338-344. [CrossRef] 
3. Piccolella, S.; Crescente, G.; Volpe, M.G.; Paolucci, M.; Pacifico, S. UHPLC-HR-MS/MS-Guided Recovery of Bioactive Flavonol Compounds from Greco di Tufo Vine Leaves. Molecules 2019, 24, 3630. [CrossRef]

4. Piccolella, S.; Bianco, A.; Crescente, G.; Santillo, A.; Chieffi Baccari, G.; Pacifico, S. Recovering Cucurbita pepo cv. 'Lungo Fiorentino' Wastes: UHPLC-HRMS/MS metabolic profile, the basis for establishing their nutra- and cosmeceutical valorisation. Molecules 2019, 24, 1479. [CrossRef] [PubMed]

5. Bujor, O.-C.; Talmaciu, I.A.; Volf, I.; Popa, V.I. Biorefining to recover aromatic compounds with biological properties. Tappi J. 2015, 14, 187-193. [CrossRef]

6. Gullón, P.; Gullón, B.; Astray, G.; Munekata, P.E.S.; Pateiro, M.; Lorenzo, J.M. Value-Added Compound Recovery from Invasive Forest for Biofunctional Applications: Eucalyptus Species as a Case Study. Molecules 2020, 25, 4227. [CrossRef] [PubMed]

7. Barjoveanu, G.; Pătrăuțanu, O.-A.; Teodosiu, C.; Volf, I. Life cycle assessment of polyphenols extraction processes from waste biomass. Sci. Rep. 2020, 10, 1-12. [CrossRef]

8. Vek, V.; Oven, P.; Poljanšek, I. Review on Lipophilic and Hydrophilic Extractives in Tissues of Common Beech. Drv. Ind. 2016, 67, 85-96. [CrossRef]

9. Hofmann, T.; Nebehaj, E.; Albert, L. The high-performance liquid chromatography/multistage electrospray mass spectrometric investigation and extraction optimization of beech (Fagus sylvatica L.) bark polyphenols. J. Chromatogr. A. 2015, 1393, 96-105. [CrossRef] [PubMed]

10. Siger, A.; Dwiecki, K.; Borzyszkowski, W.; Turski, M.; Rudzińska, M.; Nogala-Kalucka, M. Physicochemical characteristics of the cold-pressed oil obtained from seeds of Fagus sylvatica L. Food Chem. 2017, 225, 239-245. [CrossRef]

11. Cadahía, E.; de Simón, M.B.F.; Aranda, I.; Sanz, M.; Sanchez-Gomez, D.; Pinto, E. Non-targeted Metabolomic Profile of Fagus Sylvatica L. Leaves using Liquid Chromatography with Mass Spectrometry and Gas Chromatography with Mass Spectrometry. Phytochem. Anal. 2014, 26, 171-182. [CrossRef]

12. Pirvu, L.; Grigore, A.; Bubueanu, C.; Draghici, E.M. Comparative analytical and antioxidant activity studies on a series of Fagus sylvatica L. leaves extracts. J. Planar Chromatogr. Mod. TLC 2013, 26, 237-242. [CrossRef]

13. Romussi, G.; Bignardi, G.; Falsone, G.; Wendisch, D. Triterpene saponins from Fagus sylvatica L. Arch. Pharm. 1987, 320, 153-158. [CrossRef]

14. Nicu, A.I.; Pirvu, L.; Stoian, G.; Vamanu, A. Antibacterial activity of ethanolic extracts from Fagus sylvatica L. and Juglans regia L. leaves. Farmacia 2018, 66, 483-486. [CrossRef]

15. Pirvu, L.; Nicu, A.I.; Schiopu, S.; Coprean, D. Gastroprotective potential of Fagus sylvatica leaves extracts on stress-induced ulcer model on rats. Sci. Bull. Series F. Biotechnol. 2016, 20, 293-299.

16. Frédérich, M.; Marcowycz, A.; Cieckiewicz, E.; Mégalizzi, V.; Angenot, L.; Kiss, R. In Vitro Anticancer Potential of Tree Extracts from the Walloon Region Forest. Planta Med. 2009, 75, 1634-1637. [CrossRef]

17. Jehnes, S.; Betz, G.; Bahnweg, G.; Haberer, K.; Sandermann, H.; Rennenberg, H. Tree internal signalling and defence reactions under ozone exposure in sun and shade leaves of European beech (Fagus sylvatica L.) trees. Plant Biol. 2007, 9, 253-264. [CrossRef] [PubMed]

18. Pacifico, S.; Galasso, S.; Piccolella, S.; Kretschmer, N.; Pan, S.P.; Marciano, S.; Bauer, R.; Monaco, P. Seasonal variation in phenolic composition, antioxidant and anti-inflammatory activities of Calamintha nepeta (L.). Savi. Int. J. Food Res. 2014, 69, 121-132. [CrossRef]

19. Ferrara, L.; Dosi, R.; Di Maro, A.; Guida, V.; Cefarelli, G.; Pacifico, S.; Mastellone, C.; Fiorentino, A.; Rosati, A.; Parente, A. Nutritional values, metabolic profile and radical scavenging capacities of wild asparagus (A. acutifolius L.). J. Food Compos. Anal. 2011, 24, 326-333. [CrossRef]

20. Piccolella, S.; Fiorentino, A.; Pacifico, S.; D’Abrosca, B.; Uzzo, P.; Monaco, P. Antioxidant Properties of Sour Cherries (Prunus cerasus L.): Role of Colorless Phytochemicals from the Methanolic Extract of Ripe Fruits. J. Agric. Food Chem. 2008, 56, $1928-1935$. [CrossRef]

21. Wan, H.; Liu, D.; Yu, X.; Sun, H.; Li, Y. A Caco-2 cell-based quantitative antioxidant activity assay for antioxidants. Food Chem. 2015, 175, 601-608. [CrossRef]

22. Jablonský, M.; Škulcová, A.; Malvis, A.; Šima, J. Extraction of value-added components from food industry based and agro-forest biowastes by deep eutectic solvents. J. Biotechnol. 2018, 282, 46-66. [CrossRef] [PubMed]

23. Ferreira-Santos, P.; Zanuso, E.; Genisheva, Z.; Rocha, C.M.R.; Teixeira, J.A. Green and Sustainable Valorization of Bioactive Phenolic Compounds from Pinus By-Products. Molecules 2020, 25, 2931. [CrossRef]

24. Tanase, C.; Mocan, A.; Coșarcă, S.; Gavan, A.; Nicolescu, A.; Gheldiu, A.-M.; Vodnar, D.C.; Muntean, D.-L.; Crișan, O. Biological and Chemical Insights of Beech (Fagus sylvatica L.) Bark: A Source of Bioactive Compounds with Functional Properties. Antioxidants 2019, 8, 417. [CrossRef]

25. Hofmann, T.; Tálos-Nebehaj, E.; Albert, L. Leaf polyphenols as indicators of climatic adaptation of Beech (Fagus sylvatica L.)—An HPLC-MS/MS via MRM approach. Int. Labmate 2017, 42, 12-14.

26. Piccolella, S.; Crescente, G.; Candela, L.; Pacifico, S. Nutraceutical polyphenols: New analytical challenges and opportunities. J. Pharm. Biomed. Anal. 2019, 175, 112774. [CrossRef]

27. Potter, C.; Jones, D. Polyphenolic Profiling of Forestry Waste by UPLC-HDMSE. Processes 2020, 8, 1411. [CrossRef] 
28. Fernández-Agulló, A.; Freire, M.S.; Ramírez-López, C.; Fernández-Moya, J.; González-Álvarez, J. Valorization of residual walnut biomass from forest management and wood processing for the production of bioactive compounds. Biomass Convers. Biorefinery 2021, 11, 609-618. [CrossRef]

29. Gascón, S.; Jiménez-Moreno, N.; Jiménez, S.; Quero, J.; Rodríguez-Yoldi, M.J.; Ancín-Azpilicueta, C. Nutraceutical composition of three pine bark extracts and their antiproliferative effect on Caco-2 cells. J. Funct. Food. 2018, 48, 420-429. [CrossRef]

30. Trivelato, P.; Mayer-Laigle, C.; Barakat, A.; Fulcrand, H.; Aouf, C. Douglas bark dry fractionation for polyphenols isolation: From forestry waste to added value products. Ind. Crop. Prod. 2016, 86, 12-15. [CrossRef]

31. Pacifico, S.; Piccolella, S.; Nocera, P.; Tranquillo, E.; Poggetto, F.D.; Catauro, M. New insights into phenol and polyphenol composition of Stevia rebaudiana leaves. J. Pharm. Biomed. Anal. 2019, 163, 45-57. [CrossRef]

32. Gohari, A.; Saeidnia, S.; Mollazadeh, K.; Yassa, N.; Malmir, M.; Shahverdi, A. Isolation of a new quinic acid derivative and its antibacterial modulating activity. DARU J. Pharm. Sci. 2010, 18, 69-73.

33. Xiao, J.; Capanoglu, E.; Jassbi, A.R.; Miron, A. Advance on the Flavonoid C-glycosides and Health Benefits. Crit. Rev. Food Sci. Nutr. 2016, 56, S29-S45. [CrossRef]

34. Ferreres, F.; Silva, B.M.; Andrade, P.; Seabra, R.M.; Ferreira, M.A. Approach to the study of C-glycosyl flavones by ion trap HPLC-PAD-ESI/MS/MS: Application to seeds of quince (Cydonia oblonga). Phytochem. Anal. 2003, 14, 352-359. [CrossRef]

35. Rawat, P.; Kumar, M.; Sharan, K.; Chattopadhyay, N.; Maurya, R. Ulmosides A and B: Flavonoid 6-C-glycosides from Ulmuswallichiana, stimulating osteoblast differentiation assessed by alkaline phosphatase. Bioorganic Med. Chem. Lett. 2009, 19, 4684-4687. [CrossRef]

36. Zhou, Z.; Li, N.; Zhang, H.-F.; Wang, Q.-Q.; Yu, Q.; Wang, F.; Dai, Y.-H.; Wang, D.; Liu, D.-C. Simultaneous quantitative analysis of 11 flavonoid derivatives with a single marker in persimmon leaf extraction and evaluation of their myocardium protection activity. J. Nat. Med. 2019, 73, 404-418. [CrossRef]

37. Candela, L.; Formato, M.; Crescente, G.; Piccolella, S.; Pacifico, S. Coumaroyl Flavonol Glycosides and More in Marketed Green Teas: An Intrinsic Value beyond Much-Lauded Catechins. Molecules 2020, 25, 1765. [CrossRef]

38. Lai, Y.-C.; Chen, C.-K.; Tsai, S.-F.; Lee, S.-S. Triterpenes as $\alpha$-glucosidase inhibitors from Fagus hayatae. Phytochemistry 2012, 74, 206-211. [CrossRef]

39. Hong, S.S.; Jeong, W.; Kim, J.K.; Kwon, J.G.; Lee, J.Y.; Ahn, E.-K.; Oh, J.; Seo, D.W.; Oh, J.S. Neolignan inhibitors of anti-gen-induced degranulation in RBL-2H3 cells from the needles of Pinus thunbergii. Fitoterapia 2014, 99, 347-351. [CrossRef]

40. Hu, J.; Shi, X.-D.; Chen, J.-G.; Li, C.-S. Two new rhamnopyranosides of neolignans from Sanguisorba officinalis. J. Asian Nat. Prod. Res. 2012, 14, 171-175. [CrossRef]

41. Ma, Q.; Wei, R.; Yang, M.; Huang, X.; Zhong, G.; Sang, Z.; Dong, J.; Shu, J.; Liu, J.; Zhang, R.; et al. Structures and biological evaluation of phenylpropanoid derivatives from Murraya koenigii. Bioorganic Chem. 2019, 86, 159-165. [CrossRef] [PubMed]

42. Matzke, K.; Riederer, M. A comparative study into the chemical constitution of cutins and suberins from Picea abies (L.) Karst, Quercus robur L., and Fagus sylvatica L. Planta 1991, 185, 233-245. [CrossRef] [PubMed]

43. Yang, N.-Y.; Yang, Y.-F.; Li, K. Analysis of Hydroxy Fatty Acids from the Pollen of Brassica campestris L. var. oleifera DC. by UPLC-MS/MS. J. Pharm. 2013, 2013, 1-6. [CrossRef]

44. Wei, K.; Li, W.; Koike, K.; Liu, L.; Fu, X.; Lin, L.; Chen, Y.; Nikaido, T. Two New Galloylglucosides from the Leaves of Mallotus furetianus. Chem. Pharm. Bull. 2004, 35, 776-779. [CrossRef] [PubMed]

45. Li, Y.J.; Wei, H.L.; Qi, L.W.; Chen, J.; Ren, M.T.; Li, P. Characterization and identification of saponins in Achyranthes bidentata by rapid-resolution liquid chromatography with electrospray ionization quadrupole time-of-flight tandem mass spectrometry. Rapid. Commun. Mass Spectrom. 2010, 24, 2975-2985. [CrossRef] [PubMed]

46. Bisht, A.; Dickens, M.; Rutherfurd-Markwick, K.; Thota, R.; Mutukumira, A.N.; Singh, H. Chlorogenic Acid Potentiates the Anti-Inflammatory Activity of Curcumin in LPS-Stimulated THP-1 Cells. Nutrients 2020, 12, 2706. [CrossRef] [PubMed]

47. Meng, S.; Cao, J.; Feng, Q.; Peng, J.; Hu, Y. Roles of Chlorogenic Acid on Regulating Glucose and Lipids Metabolism: A Review. Evidence-Based Complement. Altern. Med. 2013, 2013, 1-11. [CrossRef]

48. Hofmann, T. Structure and Antioxidant Efficiency of Beech (Fagus sylvatica L.) Bark Polyphenols Unraveled by High-Performance Liquid Chromatography/Photodiode Array Detection/Multistage Electrospray Mass Spectrometry and Chemometrics. In Polyphenols in Plants; Watson, R., Ed.; Elsevier: London, UK, 2019; pp. 83-109.

49. Zaidieh, T.; Smith, J.R.; Ball, K.E.; An, Q. ROS as a novel indicator to predict anticancer drug efficacy. BMC Cancer 2019, 19, 1-14. [CrossRef] [PubMed]

50. Gattuso, G.; Barreca, D.; Gargiulli, C.; Leuzzi, U.; Caristi, C. Flavonoid Composition of Citrus Juices. Molecules 2007, 12, 1641-1673. [CrossRef]

51. Dini, I.; Laneri, S. The New Challenge of Green Cosmetics: Natural Food Ingredients for Cosmetic Formulations. Molecules 2021, 26, 3921. [CrossRef]

52. Wang, J.; Fang, X.; Ge, L.; Cao, F.; Zhao, L.; Wang, Z.; Xiao, W. Antitumor, antioxidant and anti-inflammatory activities of kaempferol and its corresponding glycosides and the enzymatic preparation of kaempferol. PLoS ONE 2018, 13, e0197563. [CrossRef]

53. Sharma, A.; Kashyap, D.; Sak, K.; Tuli, H.S.; Sharma, A. Therapeutic charm of quercetin and its derivatives: A review of research and patents. Pharm. Pat. Anal. 2018, 7, 15-32. [CrossRef] 
54. Hofmann, T.; Nebehaj, E.; Stefanovits-Bányai, É.; Albert, L. Antioxidant capacity and total phenol content of beech (Fagus sylvatica L.) bark extracts. Ind. Crop. Prod. 2015, 77, 375-381. [CrossRef]

55. Pirvu, L.; Armatu, A.; Bubueanu, C.; Pintilie, G.; Nita, S. Obtaining and chemical characterization of some vegetal extracts with corrosion-scaling inhibition properties. Part I. Fagus sylvatica L. and Alii cepae bulbus extracts. Rom. Biotechnol. Lett. 2010, 15, 5683-5689.

56. Bae, Y.S.; Oh, H.; Rhee, S.G.; Yoo, Y.D. Regulation of reactive oxygen species generation in cell signaling. Mol. Cells 2011, 32, 491-509. [CrossRef]

57. Song, Y.; Han, M.; Zhang, X. Quercetin suppresses the migration and invasion in human colon cancer Caco-2 cells through regulating toll-like receptor 4/nuclear factor-kappa B pathway. Pharmacogn. Mag. 2016, 12, S237-S244. [CrossRef] 